\title{
Symmetry Breaking Phenomena in Thermovibrationally Driven Particle Accumulation Structures
}

\author{
Marcello Lappa and Thomas Burel
}

Department of Mechanical and Aerospace Engineering, University of Strathclyde, James Weir Building, 75 Montrose Street, Glasgow, G1 1XJ, UK - email: marcello.lappa@strath.ac.uk

\begin{abstract}
Following the recent discovery of new three-dimensional particle attractors driven by joint (fluid) thermovibrational and (particle) inertial effects in closed cavities with various shapes and symmetries (Phys. Fluids, 26(9), 093301, 2014 and Phys. Fluids, 31(7), 073303, 2019), the present analysis continues this line of inquiry by probing influential factors hitherto not considered; among them, the role of the steady component of thermovibrational convection, i.e. the time-averaged velocity field that is developed by the fluid due to the non-linear nature of the overarching balance equations. It is shown how this apparently innocuous problem opens up a vast parameter space, which includes several variables, comprising (but not limited to) the frequency of vibrations, the socalled 'Gershuni number', the size of particles (Stokes number) and their relative density with respect to the surrounding fluid (density ratio). A variety of new particle structures (2D and 3D) are uncovered and a complete analysis of their morphology is presented. The results reveal an increase in the multiplicity of solutions brought in by the counter-intuitive triadic relationship among particle inertial effects and the instantaneous and time-averaged thermovibrational phenomena. Finally, a universal formula is provided that is able to predict correctly the time required for the formation of all the observed structures.
\end{abstract}

\section{Introduction}

Fluids with dispersed particles are governed by physical effects (and related parameters) that when varied can result in widely qualitatively and quantitatively different dynamical behaviors. The related paths of evolution can be extremely intricate and include phenomena such as synchronization (able to produce highly ordered patterns, see, e.g., Schwabe et al., ${ }^{1}$; Pushkin, Melnikov and Shevtsova ${ }^{2}$; Melnikov, Pushkin and Shevtsova ${ }^{3}$; Gotoda et al., ${ }^{4}$ ), or vice versa bifurcations and symmetry-breaking (leading to diametrically opposite effects see, e.g., Parthasarathy and Faeth, ; Ehara et al., ${ }^{6}$; Lappa $^{7}$ and references therein). These systems and the underlying mathematical aspects are of great interest from the viewpoint of fundamental science as the non-trivial interplay between particles and fluid makes them ideal targets to study how nonlinearity can produce states with a varying degree of disorder.

Beyond purely academic aspects, fluid-particle mixtures are widespread in science and engineering and widely studied in specific areas such as astrophysics (Bracco et al., ${ }^{8}$; Lappa, ${ }^{9}$ ), geophysics (Haszpra and Tél ${ }^{10}$ ), the sciences of life (Srivastava ${ }^{11}$ ), thermal and mechanical engineering (Saghir and Mohamed ${ }^{12}$; Acquaro ${ }^{13}$; Dasgupta et al., ${ }^{14}$ ), combustion, chemistry and materials science (Rao, ${ }^{15}$; Zhao et al., ${ }^{16}$; Balboa-Usabiaga and Delgado-Buscalioni ${ }^{17}$ ). With the rise in its industrial importance, in particular, the initially largely theoretical development of such a subject (mostly 
carried out with regard to turbulent fluid motion, given the pervasive presence of such flows in a number of industrial processes and applications, see, e.g., Eaton and Fessler ${ }^{18}$; Jayaram et al., ${ }^{19}$; Yuan et al., ${ }^{20}$; Luo et al., ${ }^{21}$ ) has received a continuation with new studies focusing on flows with laminar characteristics.

This new line of inquiry has been motivated by the need to identify new archetypal situations in which the non-linear effects at play in these systems could intentionally be used to exert a 'control' on the behavior and/or distribution of particles.

Due to such investigations, eventually, it has been recognized that the ability of some timedependent flows to promote particle clustering is not an exclusive prerogative of the turbulent regime (Haller and Sapsis ${ }^{22}$ ). Flows with a deterministic behavior (e.g., time-periodic convection) have indeed been found to support the formation of highly-ordered, high-resolution particle structures via the interplay of their time-dependent (but not turbulent) properties and the ability of dispersed solid matter to react to the fluctuations in the fluid velocity field with a certain time-lag or phase shift. As an example, a very interesting line of research dealing with these aspects is that originating from the discovery of one-dimensional flywheel-shaped particle structures in liquid bridges Refs. [1-4].

A justification for these peculiar behaviors can be rooted to the topological features of the fluid streamlines and the geometrical properties of the trajectories that particles describe in space. These paths are not necessarily coincident. A density mismatch can cause acceleration or deceleration of particles along the direction of the prevailing body forces, while their finite size can produce a drag of viscous nature essentially in the same direction of fluid motion. For these reasons, real particles do not conform to the behavior that would ideally be taken by inertia-less (point) particles transported as passive tracers by an underlying incompressible flow. Rather they tend to display the typical dynamics of a compressible medium, i.e. the spacing among particles can undergo significant variation in time and cause the emergence of locally depleted or concentrated areas ${ }^{2}$. In particular, what sets apart this new class of phenomena and the related patterning behavior from other similar dynamics induced by turbulent flows is the reproducibility and extremely regular (aesthetically appealing) morphology of the structures formed by particles (as opposed to the irregular aggregates induced by turbulent fluid motion). Moreover, particles self-assemble without electrostatic, magnetic or hydro-dynamically induced attraction and do not require propulsion devices such as beating flagella or cilia (as it would be the case for micro-organisms). They simply clump together without any need for adhesive or other bonding forces. This may be regarded as a distinguishing mark with respect to the other interesting categories of phenomena studied e.g., by Love and Pettit ${ }^{23}$ and by Tabakova and Zapruanov ${ }^{24}$, Wunenburger et al., ${ }^{25}$, Thomas and Gollub ${ }^{26}$, Ivanova et al., ${ }^{27}$, Kozlov et al., ${ }^{28}$.

This realization has led over the years to the establishment of an independent, elegant theoretical framework that is nowadays known as the "study of inertial particle in time-periodic flows"2-3, 29-38. All these phenomena share a similar physical interpretation, which might be seen as a common denominator underpinning this specific field. Nevertheless, there seems to be no limits to the variety 
of patterns or particle structures that can be attained or produced; notably, this richness follows from the many different variants of time-periodic flow that can be found in nature and technology. Time-periodicity might be a consequence of a bifurcation naturally leading a given flow from an initial steady state to a time-dependent regime in which a single frequency (and its multiples) are present, or be artificially imposed via the application of some external forcing such as 'vibrations' with well-defined amplitude and frequency to a non-isothermal fluid. The reader interested in the former situation may consider, e.g., the experimental results by Tanaka et al., ${ }^{39}$, Ueno et al., ${ }^{40}$, Schwabe and Mizev ${ }^{41}$, Gotoda et al., ${ }^{42}$ and Toyama et al., ${ }^{43}$. The latter condition is the main focus of the present study.

When a fluid is shaken in the presence of an imposed temperature difference, the emerging flow is known as thermovibrational convection (Gershuni and Lyubimov ${ }^{44}$; Mialdun et al., ${ }^{45}$; Lyubimova ${ }^{46}$; Bourab et al., ${ }^{47}$; Shevtsova et al., ${ }^{48,49}$, Maryshev et al., ${ }^{50}$; Lappa ${ }^{51}$; Vorobev and Lyubimova ${ }^{52}$ ).

Recently, a new category of particle attractors driven by joint (liquid) thermovibrational and (particle) inertial effects has been identified in closed cavities with various shapes and symmetries (Lappa ${ }^{53-55}$ ). The present analysis aims to integrate the information reported in those studies by considering a previously overlooked aspect, that is, the steady velocity component that this type of convection can produce in some circumstances.

As illustrated for a companion (isothermal) problem by Lappa ${ }^{56}$, the presence of a non-negligible self-generated steady fluid velocity is expected to determine symmetry breaking phenomena in otherwise perfect and highly ordered particle structures. By proposing to analyze situations where thermovibrational convection includes both non-zero time-averaged and instantaneous velocity components, we aim to develop new principles to enable increased control over the ability of particles to target desired locations depending on the prevailing (steady or oscillatory) behavior of the flow.

\section{Mathematical and Numerical Model}

In line with earlier studies on the same subject and, given the significant interest attracted by such a configuration in past works and its simplicity, we consider a differentially heated cavity with characteristic size L (in microgravity conditions, i.e. with no steady gravity, see Fig. 1). The force driving the flow of fluid and dispersed solid particles is a time-periodic form of buoyancy produced by the concurrent action of an imposed temperature difference and 'vibrations i.e. a time-periodic displacement of the overall fluid-particle mixture container.

A mathematical basis for the study of these problems is the classical Eulerian framework used for representing fluid flow combined with a Lagrangian approach to account for the motion of particles (treated as separate entities). This hybrid formulation is particularly convenient as it makes unnecessary complex mathematical manipulations and transformations. Moreover, standard solution procedures can be used for the fluid flow and internal energy equations (Sect. 3). 


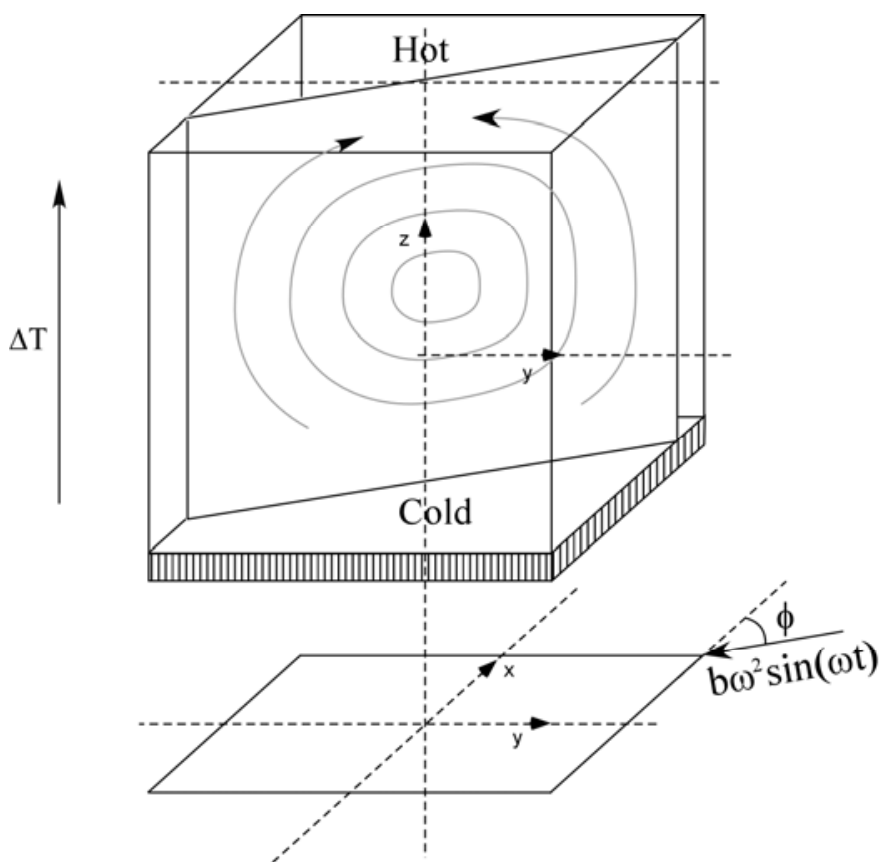

Figure 1: Cubic cavity with characteristic size L shown, delimited by solid walls (one at $\mathrm{z}=-0.5$ cooled, the other at $\mathrm{z}=0.5$ heated, adiabatic conditions on the remaining sidewalls). The vibrations are directed along the $\mathrm{y}$ axis $(\phi=\pi / 2)$ while the $\mathrm{x}$ axis plays the role of 'spanwise direction'.

\subsection{Dimensional governing equations for fluid and dispersed particles}

By indicating with $\underline{\hat{i}_{\underline{v}}}$ the unit vector relating to the direction of the fluid container displacement, with $b$ the vibration amplitude and with $\omega=2 \pi f$ the related angular frequency, imposed vibrations can mathematically be represented in a fixed laboratory frame as $\underline{s}^{\text {lab }}(t)=\operatorname{bsin}(\omega \mathrm{t}) \underline{\hat{i}_{\underline{v}}}$. From a dynamic point of view, this is equivalent to applying an acceleration varying in time as $\underline{g}(t)=g_{\omega} \sin (\omega \mathrm{t}) \hat{i}_{\underline{v}}$ where $g_{\omega}=\mathrm{b} \omega^{2}$ (formally appearing by taking the second time derivative of the displacement function). The buoyancy effect, of course, also depends on the nature of the fluid itself and the temperature distribution inside the container. Accordingly, in dimensional form the fluid transport equations for mass, momentum and internal energy can be cast in condensed form as

$\underline{\nabla} \cdot \underline{\mathrm{V}}=0$

$\rho \frac{\partial \underline{V}}{\partial t}=-\underline{\nabla} p-\rho \underline{\nabla} \cdot[\underline{V} \underline{V}]+\mu \nabla^{2} \underline{V}+\rho \mathrm{b} \omega^{2} \sin (\omega \mathrm{t}) \underline{\hat{i}_{v}}$

$\rho C_{v} \frac{\partial T}{\partial t}+\rho C_{v} \underline{\nabla} \cdot[\underline{V T}]=\lambda \nabla^{2} T$ 
where the vector $\underline{V}$ contains the variables $[u, v, w](u, v$ and $w$ being the velocity components along the $x, y$ and $z$ directions), $p$ is the pressure, $T$ is the temperature and $\rho, \mu, \lambda$ and $C_{v}$ are the fluid density, dynamic viscosity, thermal conductivity and specific heat at constant volume, respectively. This formulation clearly reflects the assumption of incompressible fluid as witnessed by the specific form taken by the balance equation for mass, it being reduced to the condition of solenoidal velocity field (mathematical condition corresponding to the constraint of fluid parcels evolving in the flow in such a way that their volume remains constant). Closure of the problem from a mathematical point of view, however, requires the introduction of an additional relationship needed to link the changes of density appearing in the momentum equation to the temperature (this being required to properly account for the buoyancy effects). By using the classical Boussinesq approximation (which has enjoyed a widespread use in past studies on both classical buoyancy and thermovibrational convection), such relationship reduces to a linear proportionality law formally corresponding to a Taylor series expansion of the density with respect to temperature truncated after its first order term $^{57}$ (while the other thermodynamic properties are generally considered constant, see, e.g., Mialdun et al., ${ }^{45}$; Lyubimova ${ }^{46}$; Bourab et al., ${ }^{47}$; Lappa $^{51}$ ).

In line with our umbrella of on-going research on this subject ${ }^{37,53-55}$, and given the success already enjoyed by this formulation in other parallel lines of research $\mathrm{h}^{2-3,30-32,58}$, we rely on a separate Lagrangian strategy to track particles. In particular, the need to account for particle inertial and drag effects (produced by the different density of particles with respect to the surrounding liquid and their non-infinitesimal size), is naturally dealt with through the so-called Maxey-Riley equation (see, e.g., Maxey and Riley ${ }^{59}$, Kuhlmann et al., ${ }^{60}$ ), which in (dimensional) vector form reads:

$\rho_{p} \frac{d \underline{V}_{p}}{d t}=\rho \frac{D \underline{V}}{D t}+\frac{9}{2} \kappa \frac{\mu}{R_{p}^{2}}\left(\underline{V}-\underline{V}_{p}\right)+\frac{\rho}{2}\left(\frac{D \underline{V}}{D t}-\frac{d \underline{V}_{p}}{d t}\right)+\left(\rho_{p}-\rho\right) \mathrm{b} \omega^{2} \sin (\omega \mathrm{t}) \hat{i}_{\underline{v}}$

where $\underline{V}_{p}=\left[u_{p}, v_{p}, w_{p}\right]$ is the particle velocity and $\kappa$ is a corrective factor used to model the departure of the particle viscous drag from the classical Stokes formula (see Sect. 2.2). In particular, the various terms appearing at the right hand side of this equation represent, respectively, the force that the undisturbed flow exerts on the generic particle, the above-mentioned viscous drag, the virtualadded mass force and the time-varying buoyancy effect due to the presence of vibrations. The socalled Basset force is neglected (the reader being referred to Ref [35] and references therein for an extensive treatment of this aspect, which is not duplicated here for the sake of brevity).

As the reader will realize by examining the mathematical shape of these terms, many of them directly depend on the velocity of the fluid, which provides the aforementioned link between the Eulerian and Lagrangian representations, i.e. between eq. (4) and eqs. (1-3).

In such a context, we also assume that particles are made of a highly heat-conducting material, so that their temperature can be assumed equal to the temperature of the ambient fluid.

The non-dimensional problem, which has been glossed over here, is put in a proper mathematical context in the next subsection. 


\subsection{Non-dimensional formulation}

Following common practice in the context of studies devoted to standard buoyancy flow (i.e. convection produced by a steady acceleration), the different factors contributing to the development of thermovibrational convection can be combined into a single non-dimensional parameter formally akin to the so-called Rayleigh number, i.e.

$R a_{\omega}=\frac{b \omega^{2} \beta_{T} \Delta T L^{3}}{v \alpha}$

where $\Delta T$ is the applied temperature difference, $\alpha=\lambda / \rho C_{v}$ is the fluid thermal diffusivity and $\beta_{T}$ is the fluid thermal expansion coefficient formally linking the fluid density to its temperature through the aforementioned Taylor series expansion (truncated in order to make the resulting relationship linear).

Given the peculiar nature of thermovibrational convection and its intrinsic property of giving rise to different regimes depending on the magnitude of vibration frequency and amplitude, however, a proper characterization of this kind of flow generally requires the introduction of three distinct nondimensional parameters, namely, the nondimensional vibration frequency $(\varpi)$, the nondimensional acceleration amplitude $(\gamma)$ and buoyancy factor $(\beta)$, defined as:

$\varpi=\frac{\omega L^{2}}{\alpha} ; \gamma=\frac{b \omega^{2} L^{3}}{\alpha^{2}} ;$ and $\beta=\left(\beta_{T} \Delta T\right)$

where obviously:

$R a_{\omega}=\gamma \beta / \operatorname{Pr}$

Explaining the dependence of this type of flow on these parameters is not as straightforward as one would imagine since a proper understanding of it does require the provision of other arguments about the ability of fluid systems, which are essentially time-periodic, to produce a non-linear stationary response.

As illustrated above, the application of vibrations to a non-isothermal fluid can be considered equivalent to imposing an acceleration varying sinusoidally in time with no average value (i.e. no residual steady acceleration). The fluid generally reacts to the application of this forcing producing a velocity field that oscillates in time with the same frequency of the vibrations. As the equations that govern the evolution of the fluid are non-linear, the synchronous behavior of the fluid with respect to the external forcing, however, can be accompanied in some specific circumstances by a different type of 'response'. 
In particular, it is known (Monti et al., ${ }^{61-62}$; Savino and Lappa ${ }^{63}$ ) that if the frequency of vibrations is relatively small and their amplitude is high, the relationship between the output (the response of the system) and the input can be assumed to be 'linear' in the sense that, as explained above, the fluid reacts to the application of an acceleration changing periodically in time with a velocity field which also evolves periodically in time (with the same frequency of the forcing). In such conditions, by taking the time-averaged value of the velocity, one would obtain a zero value. If the vibration frequency is relatively high and the amplitude small, however, a different regime can be entered (Gershuni et al., ${ }^{64}$; Gershuni and Zhukhovitskii ${ }^{65}$ ) where a significant time-averaged component of velocity is produced. This component is generally indicated as $\langle\underline{V}\rangle$ (accordingly, the related time-varying component of velocity can be defined as $\underline{V}^{\prime}=\underline{V}-\langle\underline{V}\rangle$ ).

In practice, on increasing $\varpi$ from zero, a first regime is generally met where oscillatory velocity and oscillatory temperature parts are relatively large while the time-average steady contributions can be assumed to be negligible ( $\langle\underline{V}\rangle \cong 0$ ). Conversely, at high $\varpi$ the relative importance of these terms is swapped and the time-average contributions become dominant $\left(\langle\underline{V}\rangle>>\left|\underline{V}^{\prime}\right|\right)$.

While $R a_{\omega}$ accounts for the amplitude of the time-varying component of velocity, the magnitude of the steady component can be characterized using a different non-dimensional group, generally known as the Gershuni number (Gs). This number can still be expressed as a combination of the three independent parameters $\varpi, \gamma$ and $(\beta)$, i.e. it reads:

$G s=\frac{\left(b \omega \beta_{T} \Delta T L\right)^{2}}{2 v \alpha}=\frac{\left(\beta_{T} \Delta T L\right)^{2}}{2 v \alpha}\left(\frac{g_{\omega}}{\omega}\right)^{2}=\frac{1}{2 \operatorname{Pr}}\left(\frac{\beta \gamma}{\varpi}\right)^{2}=\frac{\operatorname{Pr}}{2}\left(\frac{R a_{\omega}}{\varpi}\right)^{2}$

The presence of solid particles dispersed in the fluid obviously increases further the complexity of the overall non-dimensional problem defined above by enriching it with other characteristic parameters. These are the ratio of the particle to the fluid density

$\xi=\rho_{\mathrm{p}} / \rho$

and the particle Stokes number, defined as

$S t=\frac{2}{9} \frac{R_{p}^{2}}{L^{2}}$

where $R_{p}$ is the particle radius.

All these characteristic numbers simply reflect the physical nature of the considered problem where particle dynamics are affected by vibrations via two concurrent mechanisms, these being the direct effect of the resulting accelerations on the particles due to the existing density gap with respect to the surrounding fluid (which would be still present in an isothermal system) and the other being 
related to the way by which the flow of thermovibrational nature transports particles according to their radius and mass. As illustrated above, in turn, the latter can be further split into a 'mean' effect and an 'instantaneous' effect induced by the aforementioned periodic velocity $\underline{V}$ ' $=\underline{V}-\langle\underline{V}\rangle$ oscillating in time at the same acceleration frequency $\omega$ of the imposed inertial disturbance (g-jitter). Assuming as reference length, velocity and time, the cavity size (L), the thermal diffusion velocity $(\alpha / \mathrm{L})$ and time $\left(\mathrm{L}^{2} / \alpha\right)$, respectively and scaling the temperature by $\Delta T$, the combined set of governing Eulerian and Lagrangian equations can finally be cast in non-dimensional form as:

$\underline{\nabla} \cdot \underline{\mathrm{V}}=0$

$\frac{\partial \underline{V}}{\partial t}=-\underline{\nabla} p-\underline{\nabla} \cdot[\underline{V} \underline{V}]+\operatorname{Pr} \nabla^{2} \underline{V}+\operatorname{Pr} R a_{\omega} T \sin (\varpi t) \underline{\hat{i}_{v}}$

$\frac{\partial T}{\partial t}+\underline{\nabla} \cdot[\underline{V T}]=\nabla^{2} T$

$\frac{d \underline{V}_{p}}{d t}=\frac{1}{\xi+1 / 2}\left[-\frac{\operatorname{Pr}}{S t} \kappa\left(\underline{V}_{p}-\underline{V}\right)+\frac{3}{2} \frac{d \underline{V}}{d t}+\frac{3}{2}(\underline{V} \cdot \underline{\nabla} \underline{V})\right]+\frac{\xi-1}{\xi+1 / 2} \gamma \sin (\varpi t) \hat{\hat{i}_{v}}$

where (see, e.g., Clift et al., ${ }^{66}$ )

$\kappa=1+0.15 \operatorname{Re}_{p}^{0.687}$

and

$\operatorname{Re}_{p}=3 \sqrt{2} \frac{S t^{1 / 2}}{\operatorname{Pr}}\left|\underline{V}_{p}-\underline{V}\right|$

Assembled in this way, these equations also show the essence of the so-called one-way coupling approach adopted in the present study, i.e. the implicit assumption that the back influence of particles on the fluid can be considered negligible (mathematically represented by the lack of terms relating to $\mathrm{d} \underline{V}_{\mathrm{p}} / \mathrm{dt}$ in the fluid momentum balance equation). This means that, though we use many particles for visualization purposes (Sect. 4), the numerical simulations performed in this work are intended for dilute fluid-particle mixtures.

\section{$\underline{2.3 \text { Initial and Boundary conditions }}$}

As initial conditions for the fluid we consider:

$\mathrm{t}=0: \quad \underline{V}(\mathrm{x}, \mathrm{y}, \mathrm{z})=0, T(\mathrm{x}, \mathrm{y}, \mathrm{z})=\mathrm{z}$ 
where $\underline{V}=0$ means that the liquid is quiescent and the non-dimensional temperature follows a linear relationship increasing from $T_{\text {Cold }}=-0.5$ on the cold sidewall to $T_{\text {Hot }}=0.5$ on the other side, while the remaining walls are adiabatic. No slip conditions are set for all solid walls (fluid velocity $\underline{V}=0$ ). Particles are assumed to be distributed uniformly and with zero velocity initially (for $t=0$ ) and to undergo elastic interaction with the solid (no-slip) walls when subjected to vibrations (for $t>0$ ).

\section{The numerical method}

As indicated in the preceding sections, the problem unknowns can be reduced to two vector quantities and two scalars, these being the velocity of the fluid and of the particles and the fluid pressure and temperature, respectively. Some of the connections existing among all these variables have already been clarified in Sect. 2, where the interrelations among them have implicitly been made evident by means of relevant physical arguments and by the related governing equations put in non-dimensional form. Additional levels of coupling, however, are brought in by the implementation of the numerical method required for the solution of these equations. This is especially true for what concerns the relationship between the fluid velocity and its pressure. Properly accounting for this interdependence generally requires some manipulation of the equations for mass and momentum balance, which formally leads to split them into sub-equations and define new equations not present in the original formulation. This strategy is indeed at the root of the socalled category of projection methods. The methods originate from the realization that the vorticity of a fluid can be decoupled from pressure gradients as these gradients do not appear in the vorticity balance equation (this equation formally obtained by taking the curl of the momentum equation does not include pressure as the curl of the gradient of a scalar quantity is always zero by definition). This implies that by solving an approximated version of the momentum equation where the presence of the pressure gradient is not considered, i.e.

$$
\frac{\partial \underline{V}^{*}}{\partial t}=\left[-\underline{\nabla} \cdot(\underline{V} \underline{V})+\operatorname{Pr} \nabla^{2} \underline{V}+\operatorname{Pr} R a_{\omega} T \sin (\varpi t) \hat{i}_{\underline{v}}\right]
$$

with $\underline{V}^{*}=0$ being imposed at the walls, one gets a velocity field that, though not physical, displays the same content of vorticity that the physical velocity would.

The resulting velocity field (called "intermediate" to distinguish it from the physical one) obviously needs further numerical elaboration in order to make it realistic. This is typically implemented by correcting it with the pressure gradient previously neglected as

$\underline{V}^{n+1}=\underline{V}^{*}-\Delta t \underline{\nabla} p$

This stage is purely formal as the pressure is still unknown after $\underline{V}^{*}$ has been determined via the solution of eq. (16). In the practical implementation it must be preceded by the numerical solution 
of the elliptic equation obtained by forcing eq. (17) into the continuity equation. This artifice formally yields a new equation for the determination of the pressure:

$$
\nabla^{2} p=\frac{1}{\Delta t} \underline{\nabla} \cdot \underline{V^{*}}
$$

Once $p$ has been determined, the final (physical) velocity field can be computed using eq. (17), which implicitly leads to the conclusion that eq. (18) must be solved with homogeneous Neumann boundary conditions for the pressure at the walls, i.e. $\underline{\nabla} p \cdot \underline{\hat{n}}$ (where $\underline{\hat{n}}$ is the unit vector perpendicular to the boundary. This condition obviously stems from the need to make $\underline{V} \cdot \underline{\hat{n}}=0$ there via application of eq. (17)). Its ability to make the problem well posed from a mathematical point of view was originally assessed by Gresho and Sani ${ }^{67}$ on the basis of principles of mass and momentum conservation combined with continuity arguments. Though it is known that this simple condition might lead to erroneous numerical boundary layers in proximity to solid walls (especially for turbulent flows), it has been used for the present simulations due to the essentially laminar nature of the considered thermovibrational convective states and because the thickness of these layers $\left(l \cong(\operatorname{Pr} \Delta t)^{1 / 2}\right)$ can be considered negligible for the time integration steps considered in the present work $\left(\Delta t=\mathrm{O}\left(10^{-7}\right) \rightarrow l=\mathrm{O}\left(10^{-3}\right)\right.$ ) (the reader interested in more sophisticated variants is referred to the existing literature, e.g., the relevant studies by Karniadakis et al., ${ }^{68}$ and Petersson ${ }^{69}$ where more accurate pressure boundary conditions were derived for high-order-in-time integration schemes; these alternate conditions typically enforce the normal derivative of the divergence to be zero on the boundary by coupling the normal derivative of the pressure to the normal component of the curl of the vorticity).

Though the modus operandi at the root of these projection methods may apparently look heuristic, in reality, it relies on a precise mathematical theorem, namely, the so-called Hodge decomposition theorem (see, e.g., Ladyzhenskaya ${ }^{70}$ ). This theorem states that any vector field can always be split into a vector part of given divergence and a second part given by the gradient of a scalar potential function. Additional insights into this category of methods and related theoretical pre-requisites or implications can be found in various works appearing in the literature (see, e.g., Brown et al., ${ }^{71}$; Armfield and Street ${ }^{72}$; Guermond et al., ${ }^{73}$ ).

In general, special care must be taken to make sure that the coupling of velocity and pressure is well-posed from a numerical point of view. This is typically achieved by implementing a staggered arrangement for these quantities ${ }^{74-75}$.

Before moving to the next section, it is also worth briefly discussing the coupling between the two vector unknowns involved in the problem, namely, the velocity of the fluid and the velocity of the particles. These two velocity fields follow different principles, as one relates to a fixed (uniform) grid covering the entire physical domain, whereas the latter is intimately associated to the positions instantaneously occupied by the particles. For the present simulations the required coupling (i.e. the reconstruction of the fluid velocity at the position occupied by the particles) has been achieved on the basis of linear interpolation schemes by which the velocity at the particle position is determined 
as a function of the velocity at the nodes (four in 2D, eight in 3D) defining the vertices of the computational cell where the particle is contained. The grids used in the present study have been based on the mesh refinement study conducted by Lappa ${ }^{53}$ and reflect the (known) fortunate absence of boundary layers in thermovibrational flow for the range of values of the Gershuni number considered in the present work (accordingly, 80 grid points along each spatial direction have been employed).

Due to the significant computational cost being anyway associated with this approach (resulting from the need to keep the time integration step extremely small especially for the case of high $\varpi$ ), part of the explorative simulations presented in Sect. 4 have been conducted in a two-dimensional framework. Two-dimensional simulations have the potential to offer valuable insights in this regard when situations are considered for which vibrations are perfectly perpendicular to the imposed temperature difference and the walls of the container (as shown in Fig. 1).

Given the unavailability of results produced by other authors on the same subject considered in the present work, the overall theoretical architecture illustrated in the present and preceding sections has been validated through comparison with the outputs of simulations conducted using different computational platforms (for the same circumstances considered in the present work, see Sect. 4). As independent software, in particular, we have considered both Open-Foam and ANSYS Fluent and related one-way-coupling particle-tracking kernels. The results have displayed excellent agreement in terms of shape, size and formation time of the emerging particle structures.

\section{Results}

In previous studies ${ }^{37,53-55}$ the Gershuni number was set to a relatively small value $\left(10^{3}\right)$ in order to filter out the disturbing presence of the self-generated steady component of velocity of thermovibrational flow, expected to interfere in a detrimental way with the delicate synchronization mechanisms leading to the emergence of particle structures. Those parametric investigations were therefore carried out for fixed values of $G s$ and $\beta$ by keeping constant the ratio $\gamma / \varpi$ (related to $G s$ via a quadratic relationship, as shown by eq. (7)). Here we intentionally target the removal of all such constraints.

More specifically, the principal objectives of the present study are: (i) the analysis of the hierarchy of symmetries displayed by the particle structures as a function of governing parameters that can be varied independently, (ii) an assessment of the (non-dimensional) size and the time required for their formation, (iii) discerning the cause-and-effect relationships responsible for the observed behaviors.

As accounting for all the influential factors described in the preceding pages ( $\left.\operatorname{Pr}, R a_{\omega}, \gamma, \varpi, S t\right)$ at the same time would lead to a 5-dimension space of variables (potentially making the interpretation of results rather difficult), we resort to the widespread procedure (see, e.g., Lappa ${ }^{35}$ ) relying on the variation of one parameter, while the others are being kept fixed. Put simply, this corresponds to an 
exploration of the space of parameters proceeding along an articulated path, which consists of mutually perpendicular (in the 5-dimension space) segments.

For simplicity we concentrate on only two values of the Prandtl number (namely $\mathrm{Pr}=6.11$ and $\operatorname{Pr}=15.5$, the former corresponding to water at $25{ }^{\circ} \mathrm{C}$, the latter being representative of $1 \mathrm{cs}$ silicone oil around $25{ }^{\circ} \mathrm{C}$ or ethanol around $30{ }^{\circ} \mathrm{C}$, respectively), a fixed value of the particles Stokes number $\left(\mathrm{St}=5 \times 10^{-6}\right)$ and allow all the other influential factors to span relatively wide ranges, i.e. $\mathrm{O}\left(10^{4}\right) \leq R a_{\omega} \leq \mathrm{O}\left(10^{6}\right), \mathrm{O}\left(10^{7}\right) \leq \gamma \leq \mathrm{O}\left(10^{9}\right), \mathrm{O}\left(10^{3}\right) \leq \varpi \leq \mathrm{O}\left(10^{4}\right)$ and $0.1 \leq \xi \leq 2.0$.

Before embarking into the description of the present results, we wish to put some emphasis on the fact that the present study was motivated by a recent companion work ${ }^{56}$ dealing with collections of inertial particles suspended in an isothermal viscous fluid and subjected to oscillatory shear (no thermal effects). Starting from a ordered distribution of particles concentrated in an area having circular shape (initial conditions corresponding to a particle-dense 'ball') and repeating several cycles of shear in time, it was found that while in the absence of non-linear time-averaged effects the set of particles could behave as a cohesive entity for long times (being stretched periodically but maintaining a sharp boundary between its particle-filled interior and the clear fluid outside), the initial ball was quickly turned into a completely disordered distribution of particles when a steady component of velocity was present in the fluid.

Because of this, but bearing in mind that the motivations underlying the present study are different (here we explore the opposite effect, that is, ability of the considered flow to produce accumulation structures starting from a dispersed distribution, i.e. the existence of 'attractors'), we examine regimes where time-averaged flow is expected to be significant and break the spatio-temporal symmetry of the flow, that is, circumstances for which $G s \geq \mathrm{O}\left(10^{3}\right)$. As anticipated at the end of Sect. 3, we apply our framework to different showcases for both three- and two-dimensional convection.

\subsection{Water-particles mixtures}

\subsubsection{Influence of the acceleration amplitude}

As a first step towards the removal of the limitations affecting earlier studies on this specific subject, first we assess the evolutionary modifications undergone by the particle structures as the nondimensional acceleration amplitude $(\gamma)$ is varied while keeping constant the non-dimensional frequency and the Rayleigh number. Following a logical approach, in particular, we start from the situation in which the Gershuni number is relatively small (circumstances for which, as shown in previous studies, the mirror symmetry of the attractors with respect to the mid-plane perpendicular to the direction of vibrations should be preserved) and then move to cases when this parameter is increased by one order of magnitude or more.

Along these lines, a first set of results is summarized in Fig. 2 assuming water as working fluid, particle-to-fluid density ratio $\xi=2, R a_{\omega}=10^{5}$, and non-dimensional angular frequency $\varpi=10^{4}$,

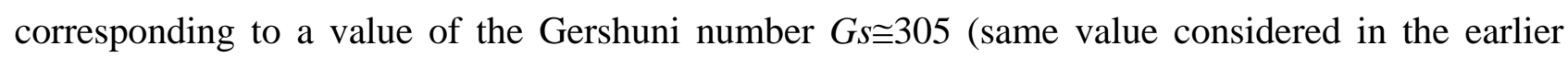


analysis by Lappa ${ }^{37}$ ). In Lappa ${ }^{37}$, very regular particle structures (consisting of two main tubular surfaces aligned along a direction perpendicular to the imposed temperature difference and to the direction of vibrations) were found to be the result of a delicate synchronization mechanism involving the purely time-periodic nature of the carrier flow (with negligible steady component) and the inertial response of non-isodense particles. As a fleeting glimpse into Fig. 2 would confirm, the spatial symmetry property with respect to the central plane $\mathrm{xz}$ is indeed retained for such a value of the Gershuni number (the emerging structures always look like 'twins' over the entire interval of values of $\gamma$ examined).

Interesting changes, however, can be identified if an assessment of the spatial extension of structures is considered. Such variations, quantitatively substantiated by the data plotted in Fig. 3, indicate that the ability of the peculiar mechanism leading particle to concentrate in regions of limited extent (originally described in Refs $[37,53]$ ) can significantly be increased (for a fixed type of particles and given values of $R a_{\omega}$ and $\varpi$ ) by making the amplitude of the acceleration higher.

Most conveniently, as shown in Fig. 3, the morphological evolution of the particle attractors can easily be characterized by introducing a distinction between the scaling laws for the vertical and horizontal size of the emerging structures. While the former displays an almost linear dependence on $\gamma$, for the latter the functional relationship is hyperbolic (as a focused cross-comparison of Figs. 3a and $3 \mathrm{~b}$ would immediately reveal).
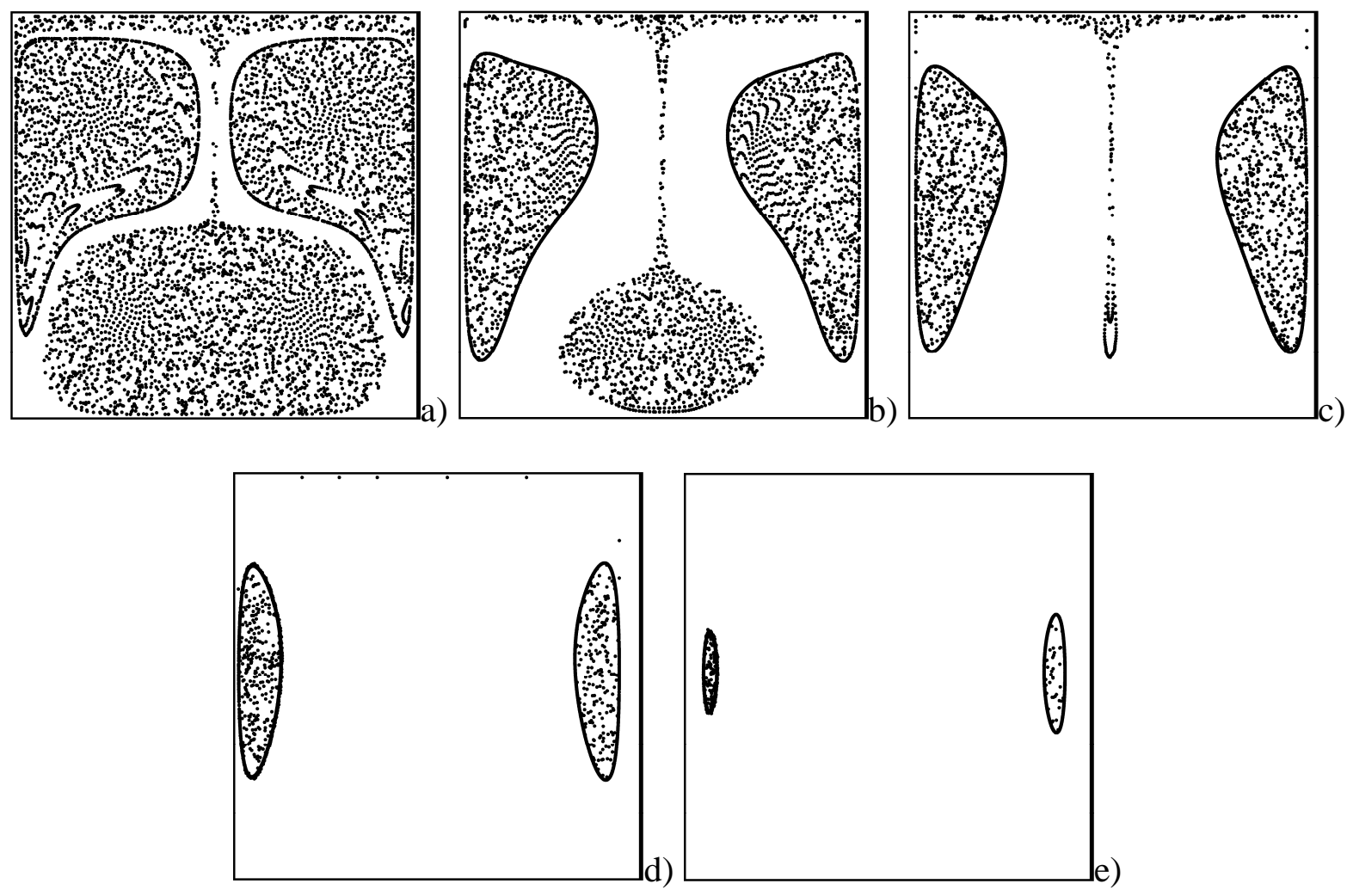

Fig. 2: Snapshots of particle structures in water for $R a_{\omega}=10^{5}, \varpi=10^{4}$ ( $G s \cong 305$ ) and $\xi=2$ for different values of the acceleration amplitude $\gamma$ (yz plane, hot side on the top, cold side on the bottom, nondimensional time $\mathrm{t} \cong 27.5$, 2D computations): a) $\gamma=10^{7}$, b) $\gamma=5 \times 10^{7}$, c) $\gamma=10^{8}$, d) $\gamma=2.5 \times 10^{8}$, e) $\gamma=5 \times 10^{8}$. 

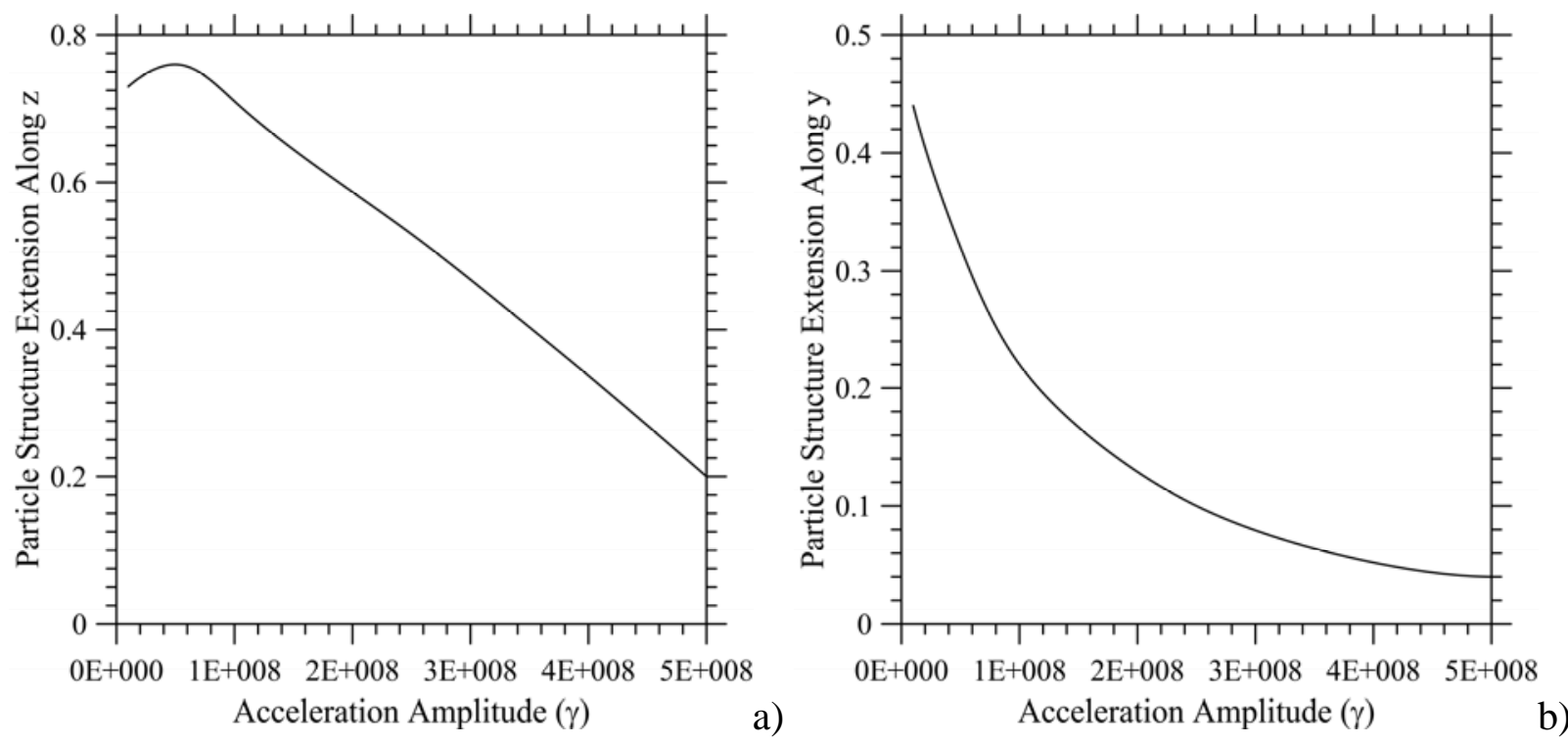

Figure 3: Spatial extension of particle structures along the $\mathrm{z}(\mathrm{a})$ and $\mathrm{y}$ (b) axes as a function of the

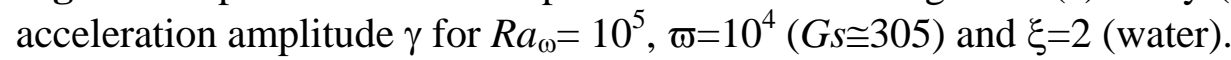

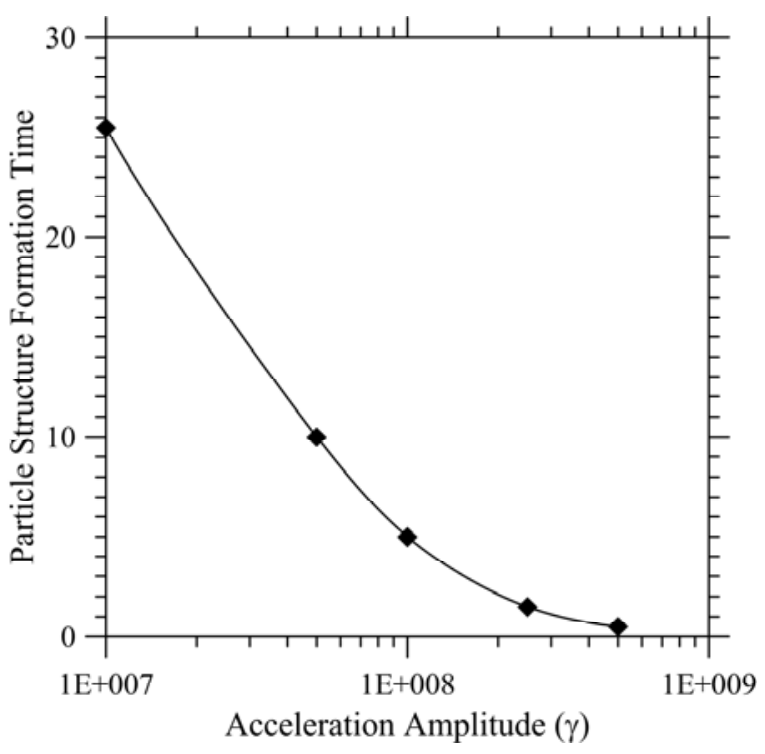

Figure 4: Particle structure formation time as a function of the acceleration amplitude for $R a_{\omega}=10^{5}$,

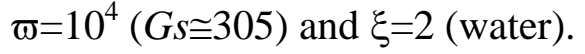

Figure 3a is also instrumental in making evident what could be interpreted as a 'saturation effect', i.e. the inability of the structures to grow further in the vertical direction when the non-dimensional acceleration amplitude drops below a certain value $\left(\gamma<10^{8}\right)$. We can readily see the physical significance of this finding by highlighting that this saturation simply follows from geometrical constraints, i.e. the existence of walls limiting the structures from below and from above along the $\mathrm{z}$ direction. Once the vertical size of the structure becomes comparable to the distance between the walls (namely the cavity size), a further decrease in $\gamma$ can no longer influence such a trend, which explains why a plateau condition is attained. 
The characterization of the considered phenomena from a spatial point of view (supported by Figs. 2 and 3 ) is complemented by the data about the temporal behavior reported in Fig. 4.

In the present work, in particular, we introduce a more rigorous interpretation of the so-called 'structure formation time' with respect to Ref [37], which we define precisely as 'the minimum time required for the formation of a closed circuit of particles'. According to the trend visible in Fig. 4, this quantity behaves as a decreasing function of the non-dimensional acceleration amplitude (for fixed values of $R a_{\omega}$ and $\varpi$ ).

Interestingly, further insights into the considered phenomena from a temporal standpoint can yet be derived from Fig. 2. Besides quantitative aspects about the spatial extension of the main particle attractors, that figure is very interesting also for another reason. The snapshots appearing there have been taken for a fixed non-dimensional time $(\mathrm{t} \cong 30)$ and they show that when values of $\gamma<10^{8}$ are considered a third region dense of particles exists in the yz plane.

This region (very evident in Fig. 2a and 2b and barely appreciable in Fig. 2c due to its limited extent) is located halfway between the two main attractors. Unlike the other two structures, its horizontal and vertical extensions are comparable (it almost takes the shape of a ball in Fig. 2b). Another important distinguishing mark is represented by the lack of a particle dense line separating it from the clear fluid outside.

Along these lines, as an aside, it is worth recalling (the reader being referred to the physical interpretations elaborated by Refs [37,53]) that the dense (black in the figures) lines corresponding to the external boundary of the two main mirror symmetric structures are a direct consequence of the specific mechanism responsible for the particle accumulation phenomena.

As already explained at the beginning of Sect. 4, this forming mechanism relies on the interplay between inertial and thermovibrational convective effects (as witnessed by the suppression of structures when one of these two mechanisms is disabled ${ }^{37,53}$ ). The specific shape taken by the mirror symmetric structures however also depends on the repetitive collisions which take place between the accumulation zones and the solid walls perpendicular to the direction of vibrations. As a result of such collisions, particles collectively driven in an area of limited extent by inertial and convective effects are forced to accumulate along the direction of the solid boundary. This line of accumulation is then folded due to the vorticity present in the fluid until a closed circuit is formed. The final closed line formed by virtue of this process apparently behaves as a barrier encapsulating all the particles that can no longer leave the attractor.

The situation for the aforementioned third intermediate region of accumulation is relatively different. The absence of a dense line delimiting its external boundary clearly indicates that the interaction with the solid walls plays no role in this case. Moreover, in Fig. 2 it is not present in circumstances for which the formation time (Fig. 4) is much smaller. In practice, by prolonging the numerical simulations to higher times, we could verify that this extra region should be regarded as an unstable accumulation area, i.e. a reservoir from which particles are progressively transferred as time increases to the two main (left and right) stable attractors adjacent to the walls perpendicular to vibrations. 


\subsubsection{Density ratio effects}

The next step of this analysis hierarchy would obviously require an assessment of the role played in all such dynamics by the relative density of particles with respect to the surrounding fluid, i.e. their inertia. The outcomes of such simulations are collected in Fig. 5. For simplicity, we focus on the same non-dimensional angular frequency and the same value of $R a_{\omega}$ already assumed for Figs. 2-4 and $\gamma=5 \times 10^{8}$ (corresponding to Fig. 2e for $\xi=2$ ).
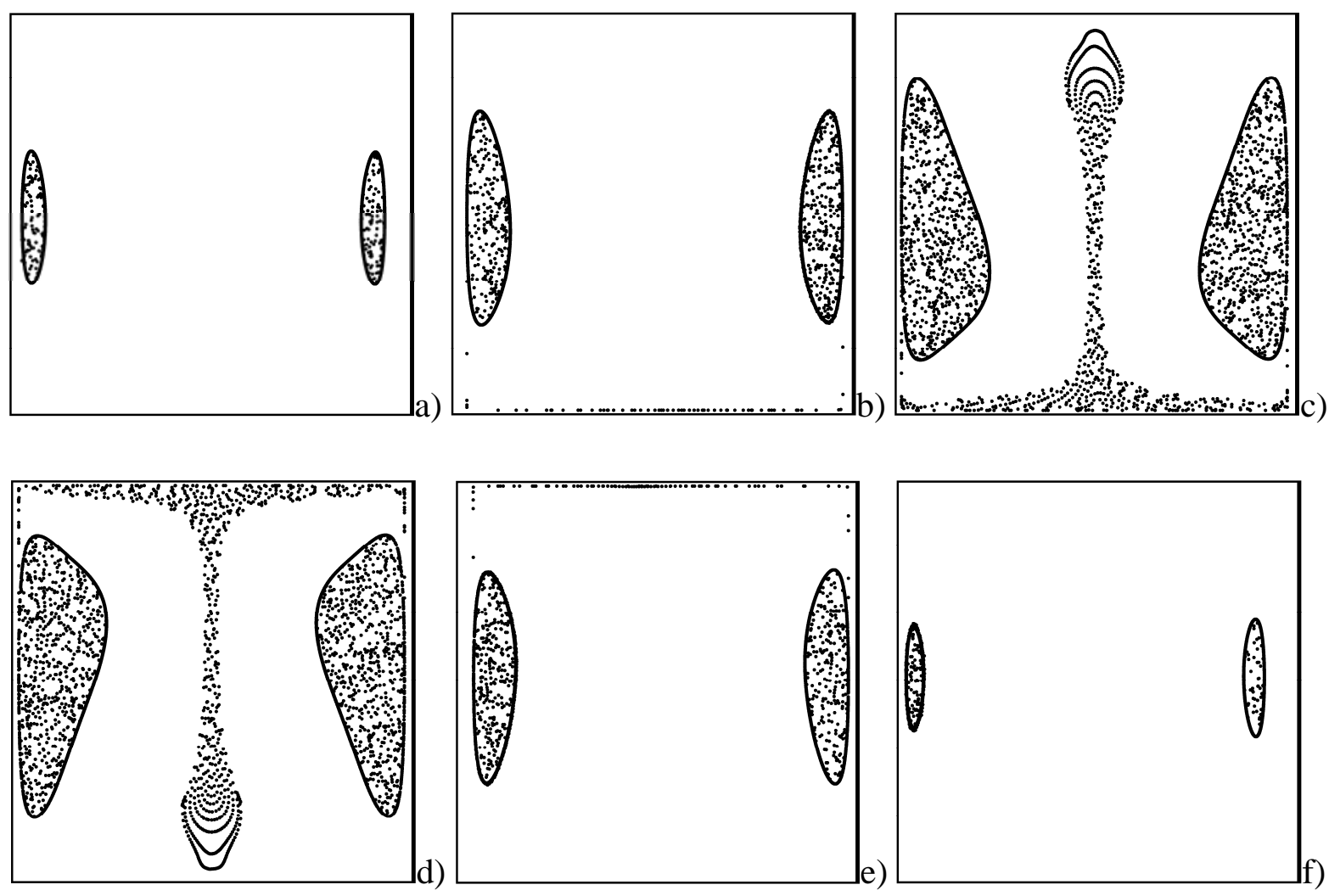

Figure 5: Snapshots of particle structures in water for $\gamma=5 \times 10^{8}, R a_{\omega}=10^{5}, \varpi=10^{4}(G s \cong 305)$ and different values of $\xi$ (yz plane, hot side on the top, cold side on the bottom, non-dimensional time $\mathrm{t} \cong 13.75$, 2D computations): a) $\xi=0.1$, b) 0.5 , c) 0.8 , d) 1.2 , e) 1.5 , f) 2.0 .

As witnessed by the ordered sequence of snapshots (for increasing values of the density ratio $\xi$ ) and the corresponding quantitative data reported in Fig. $6 \mathrm{a}$ and $6 \mathrm{~b}$, for fixed $\gamma, \varpi$ and $R a_{\omega}$, the size of the attractors scales with the quantity $|\xi-1|$. The larger this absolute value, the smaller the size of the emerging structures and vice versa. Like the dependence on $\gamma$, also in this case the functional relationships (scaling laws) change according to whether the vertical or horizontal extension of the structures is considered (the trends being approximately linear and parabolic, respectively).

As expected, the plots giving the size as a function of $\xi$ consist of two well-separated branches for particles lighter and heavier than the fluid, respectively. This marked separation is due to the wellknown suppression of the structures when particles and fluid are iso-dense ${ }^{36}$. We will come back to this important concept later; rather, here we concentrate on the interpretation of the non-negligible 
asymmetry that can be identified in the plots collected in Fig. 6 when the left and right branches are carefully compared.

Though the plots look almost mirror symmetric with respect to the condition $\xi=1$, indeed an appreciable difference can be seen especially when the two situations $\xi=0.5$ and $\xi=1.5$ (formally corresponding to the same value of the quantity $|\xi-1|$ ) are considered. We argue that this difference is the result of particle drag and added mass effects, which obviously do not obey the symmetry introduced in the problem by its dependence on $|\xi-1|$. In other words, if these effects could somehow be filtered out, the opposing branches would be perfectly symmetric (among other things, this tendency is also evident in the snapshots grouped in Fig. 5, which show that the pattern for a certain value of $\xi$ may be seen (approximately) as the reflection with respect to the horizontal direction of that obtained for $\xi-1$ ).
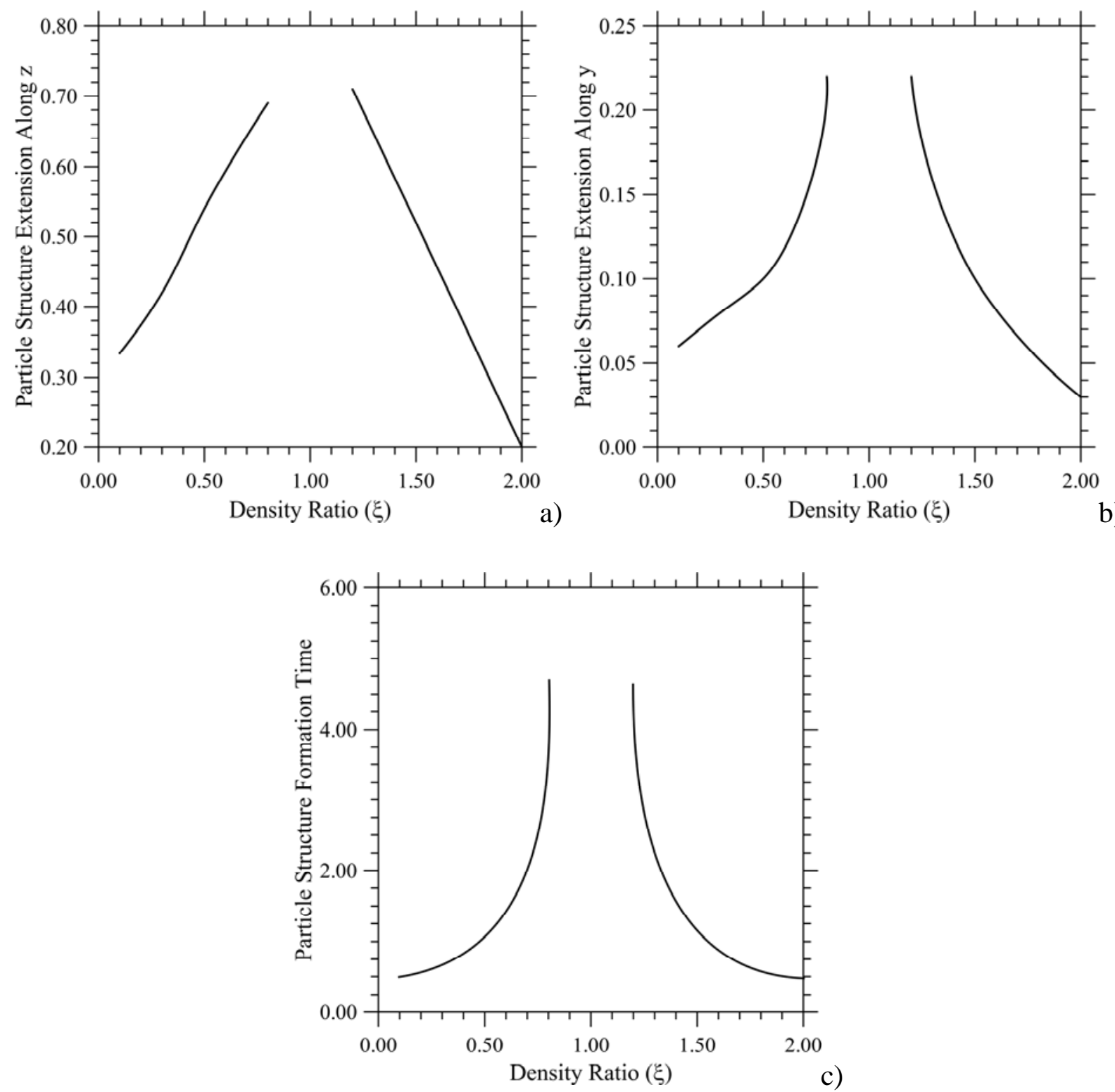

Fig. 6: Spatial extension of particle structures ((a) and (b)) and related formation time (c) as a

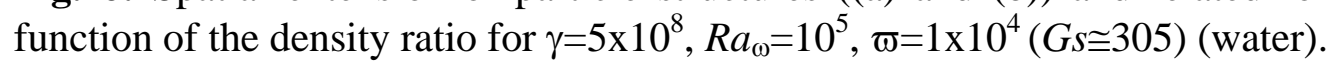


As the reader will easily realize by taking a look at Fig. 6c, the formation time also displays a similar behavior. This figure is useful also because it shows that the slope of the two opposing branches becomes very steep in the central region, this being consistent with the formation time tending to infinite in the limit as $\xi \rightarrow 1$ (as already pointed out before, owing to the hybrid inertialthermovibrational nature of these phenomena, the mechanism responsible for the formation of structures ceases to be operative if one of these two effects is lost ${ }^{37,53}$ ).
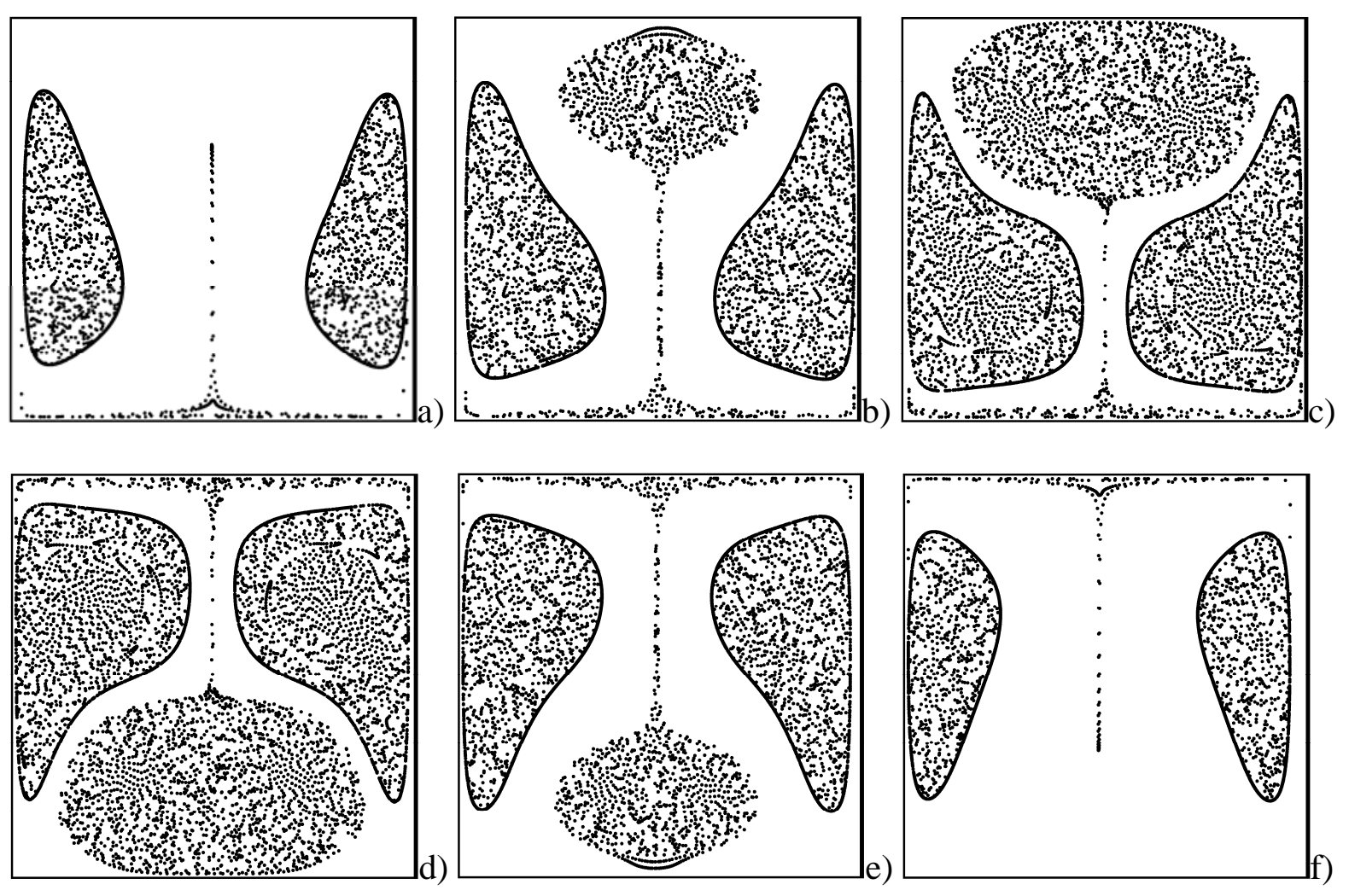

Figure 7: Snapshots of particle structures in water for $\gamma=1 \times 10^{8}, R a_{\omega}=10^{5}, \varpi=5 \times 10^{3}(G s \cong 1220)$ and different values of $\xi$ (yz plane, hot side on the top, cold side on the bottom, non-dimensional time $\mathrm{t} \cong 10,2 \mathrm{D}$ computations): a) $\xi=0.1$, b) 0.5 , c) 0.8 , d) 1.2 , e) 1.5, f) 2.0 .

Interestingly, on decreasing both $\gamma$ and $\varpi$ while keeping the Rayleigh number constant $\left(R a_{\omega}=10^{5}\right)$, the size of the structures undergoes a remarkable increase (the reader may compare Figs. 7 and 5). This explain why the aforementioned saturation (or boundary constriction) effect now starts to exert an influence on the dynamics in appreciable way. This clearly can been noticed in Fig. 8a where such mechanism causes a bending in the branches and, accordingly, a departure from the approximately linear dependence on $|\xi-1|$ seen in Fig. 6a. 
Accepted for publication in the Physics of Fluids (ISSN: 1070-6631) on 06 May 2020
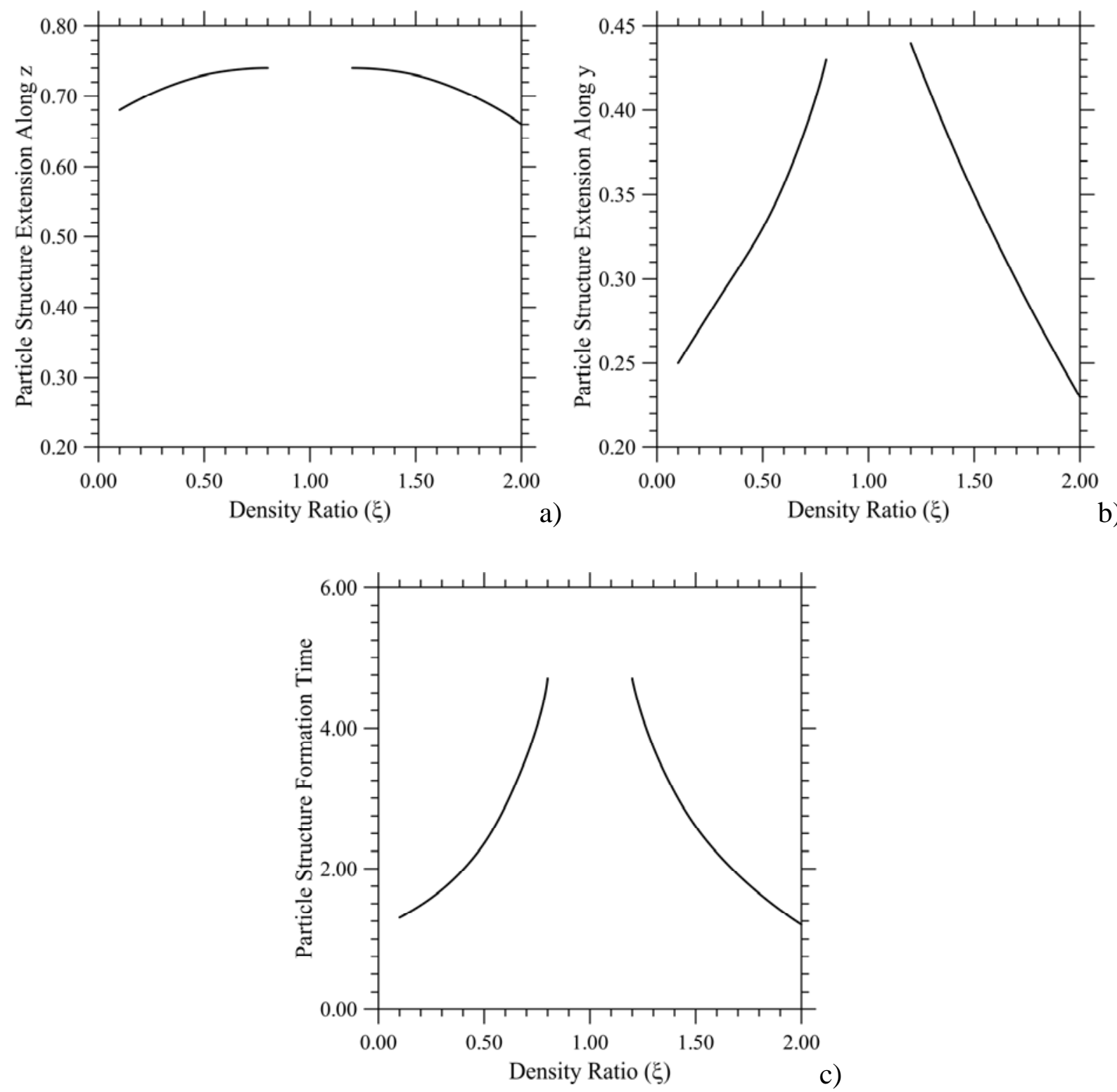

Figure 8: Spatial extension of particle structures ((a) and (b)) and related formation time (c) as a

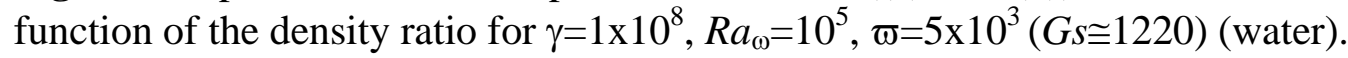

As a final look at Fig. 8c would indicate, however, the formation time remains almost unchanged (compare Fig. 8c and 6c), which leads to the conclusion that significant changes (at least one order of magnitude) of $\gamma$ are required to produce appreciable modifications of the formation time.

\subsubsection{Non-negligible time-averaged effects}

In order to explore the influence of $\varpi$ filtering out that of $\gamma$, we have performed additional simulations for the case of water at fixed $R a_{\omega}=10^{5}$, for $\gamma=1 \times 10^{8}$ and a smaller value of the angular frequency of vibrations $\left(\varpi=1 \times 10^{3}\right)$.

The outcomes of these simulations (2D cases), shown in Fig. 9, are extremely instructive. Indeed, dramatic changes can be seen there in terms of symmetry of the particle pattern. 

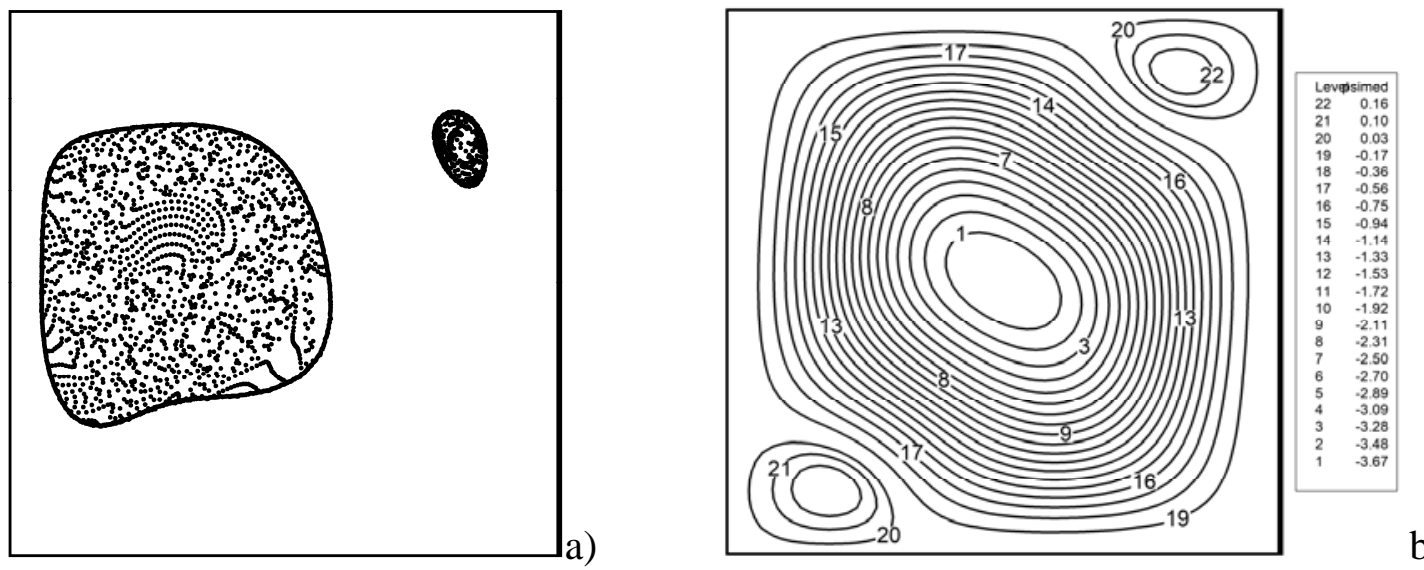

b)

Figure 9: $2 \mathrm{D}$ computations for $\gamma=1 \times 10^{8}, R a_{\omega}=10^{5}, \varpi=1 \times 10^{3}$ and $\xi=2$ (water): a) snapshot of particle structures (non-dimensional time $\mathrm{t} \simeq 0.5$ ), b) streamlines of the time-averaged velocity field $\left(G s \cong 3.05 \times 10^{4}\right)$.
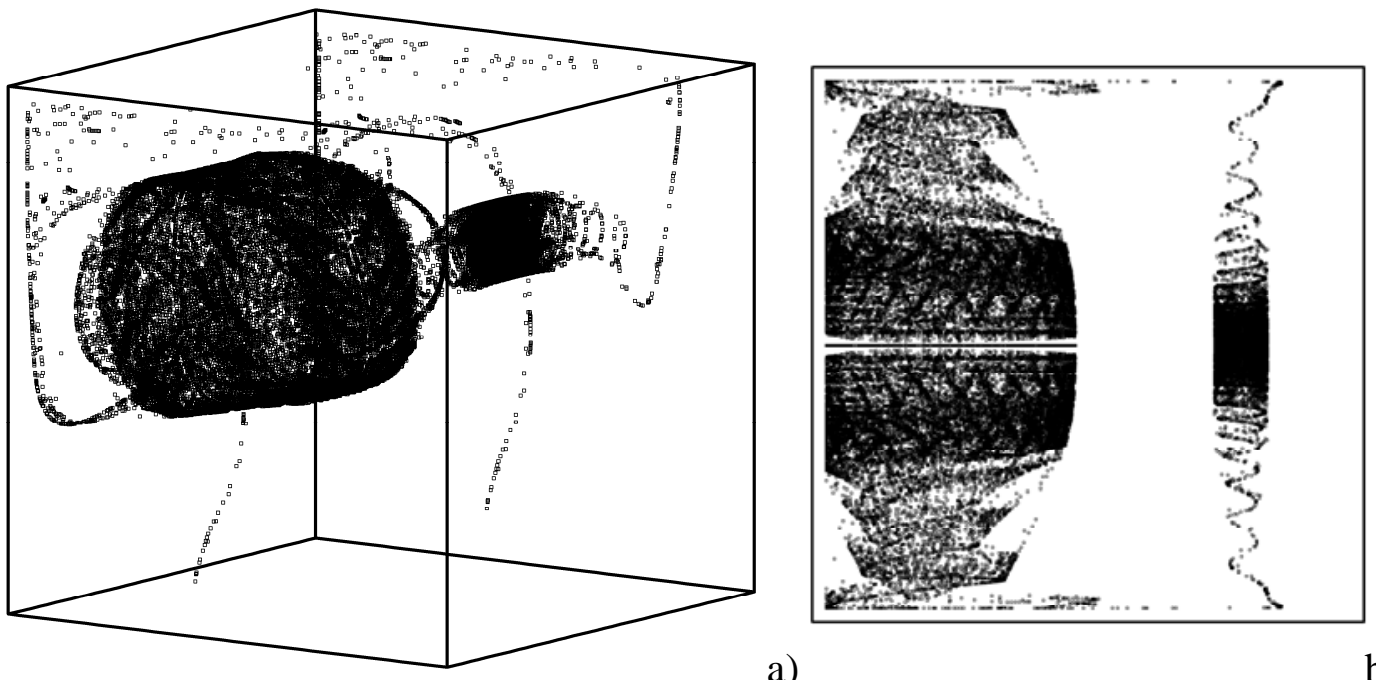

a)

b)

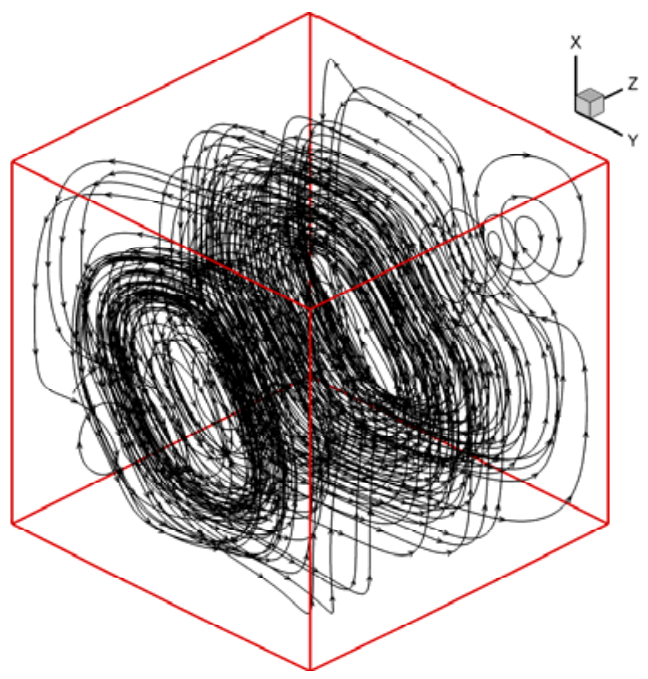

c)

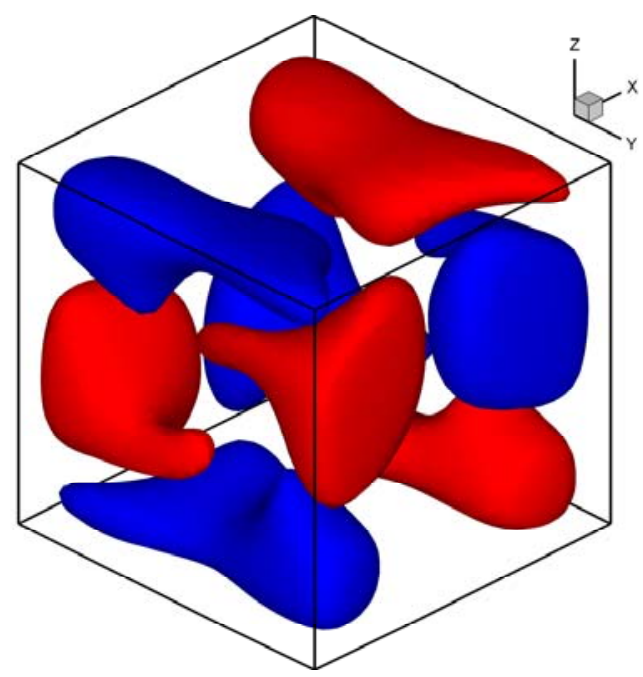

d)

Figure 10: $3 \mathrm{D}$ simulations for $\gamma=1 \times 10^{8}, R a_{\omega}=10^{5}, \varpi=1 \times 10^{3}$ and $\xi=2$ (water): a) snapshot (3D view)

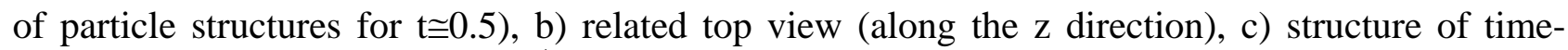
averaged flow field $\left(G s \cong 3.05 \times 10^{4}\right)$, d) isosurfaces of time-averaged velocity component along the spanwise direction x (blue level: -0.4, red level: 0.4). 
Two disconnected accumulation loci can still be identified (Fig. 9a). Nevertheless, the difference in the shape and size of the left and right structures becomes extremely evident. The area of one of the two attractors is only a relatively small fraction of the other.

In practice, the key to unlocking the puzzle about the dramatic rupture of the symmetry occurring in this case lies in the proper interpretation of the underlying fluid-dynamic process. Indeed, the loss of mirror symmetry in this case must not be ascribed to particle-related effects or a change in the synchronization mechanism responsible for the existence of attracting regions; rather it should be regarded as a natural consequence of the evolution of the velocity field.

As indicated in the figure caption, the value taken by the Gershuni number for the considered conditions is of $\mathrm{O}\left(10^{4}\right)$. For such a magnitude of Gs, mean (time-averaged) steady convection is expected to be important. Indeed, its effect can be appreciated by taking a look at Fig. 9b, where the streamlines of the time-averaged velocity field have been plotted. These streamlines clearly lack symmetry with respect to the central $(y=0)$ plane. The flow field consists of one main circulation and two small counter-rotating cells with a weaker intensity confined to the corners of the enclosure (one-main-vortex regime, corresponding to the combination of antisymmetric-antisymmetric and symmetric-symmetric convective modes, Khallouf et al., ${ }^{77}$; Lappa ${ }^{78}$ ).

This peculiar circulation system may be seen as an additional mechanism (existing in parallel with the inertial one producing the accumulation of particles illustrated by Ref [37]) able to transfer progressively particles from the area of influence of one attractor (the right one in Fig. 9a) to that of the other (the left attractor in Fig. 9a).

The effective overall morphology of the particle structures in the 3D case can be seen in Fig. 10a and 10b. We have also reported the corresponding 3D structure of the time-averaged flow in terms of streamlines (Fig. 10c) and isosurfaces of the velocity components along $\mathrm{x}$ (spanwise direction, Fig. 10d). While Fig. 10c can be used to demonstrate that the peculiar flow configuration with one main roll and two satellite vortices established in the central yz plane is maintained also in other planes parallel to it, interestingly, Fig. 10d indicates that a significant time-averaged velocity component is also effective along the $x$ axis. Remarkably, this component can be used to explain the visible 'compression' undergone by particle structures in the spanwise direction (Fig. 10b). In earlier works ${ }^{37}$, particles structures were found to extend continuously from the wall located at $\mathrm{x}=$ 0.5 to the other at $x=0.5$. In the present case, a gap of clear (or particle-depleted) fluid can be noticed (Fig. 10b) that separates the main structures from the two walls located at $x= \pm 0.5$.

In particular, this gap is much more pronounced for the particle structure that displays smaller spatial extension in the yz plane (i.e. the right structure in Figs. 10a and 10b). In order to justify this scenario, we have superposed in Fig. 11 the isosurfaces of the time-averaged velocity component in the spanwise direction and the particle distribution. As the reader will realize by inspecting this figure, the smaller particle structure is almost perfectly encapsulated between the two 'balloon' regions where this component takes appreciable values. The change in color (from red to blue) indicates that this component changes sign and it is always directed from the external walls at $\mathrm{x}=$ 0.5 and $x=0.5$ towards the mid-plane $y z$ (the reader being also referred to the sketch in Fig. 11c). 
This provides the required interpretation or explanation for the compressive or 'squeezing' effect being operative along the spanwise direction.
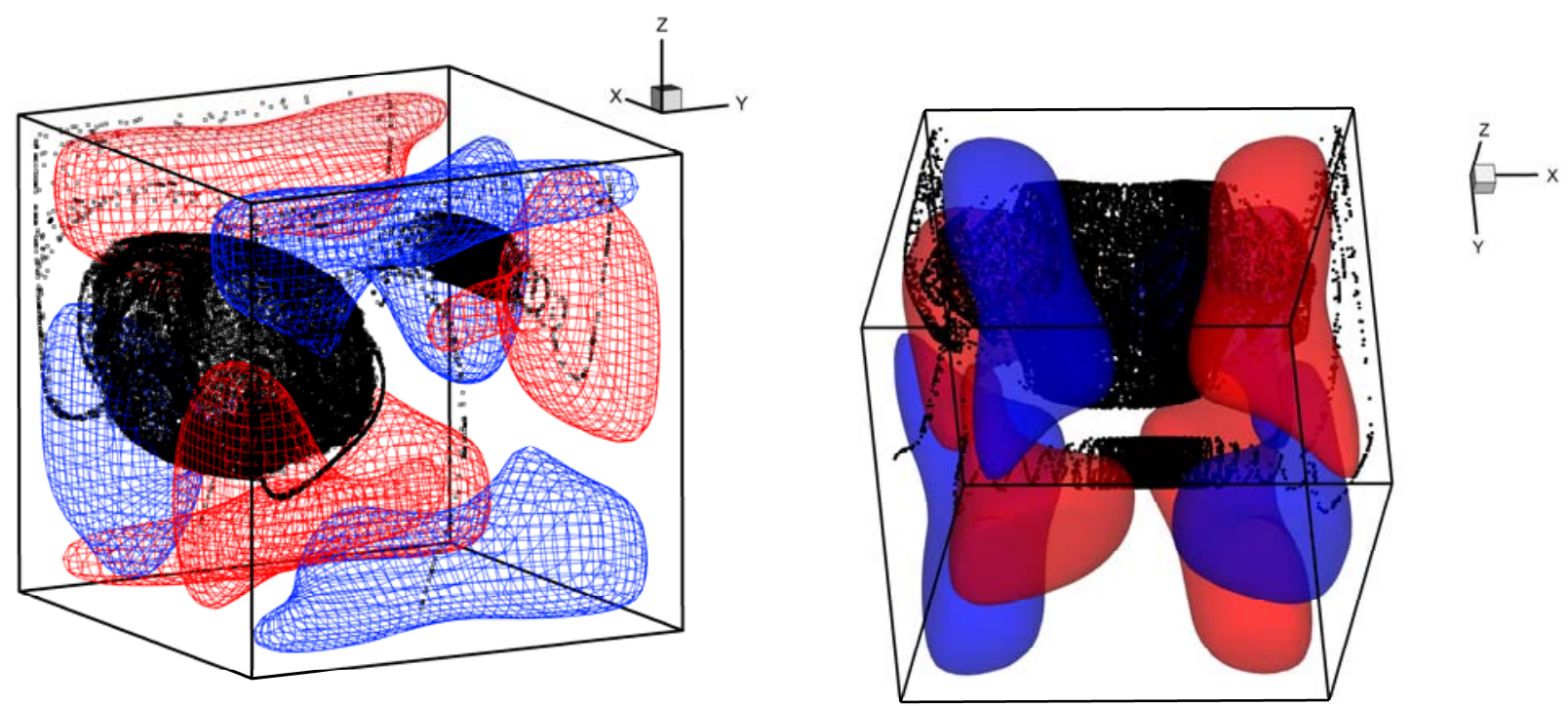

a)

b)

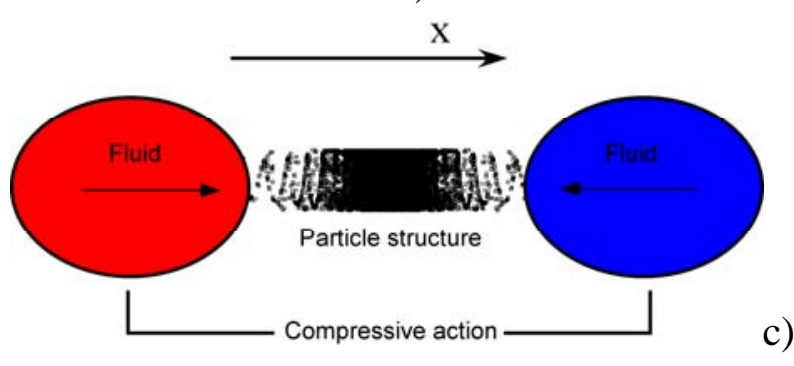

Figure 11: Isosurfaces of time-averaged velocity component along the spanwise direction (blue level: -0.4, red level: 0.4) and particle structures for $\operatorname{Pr}=6.11$ (water), $\gamma=1 \times 10^{8}, R a_{\omega}=10^{5}$ and $\varpi=1 \times 10^{3}\left(G s \cong 3.05 \times 10^{4}\right)$ : a) perspective perpendicular to the yz plane, b) perspective perpendicular to the xy plane, c) sketch showing the compressive action exerted by the clear fluid on the particle structure with smaller size.

A couple of differently colored regions (elongated balloons) can also be seen in proximity to the top and bottom walls (at $\mathrm{z}= \pm 0.5$, see Figs. $11 \mathrm{a}$ and $11 \mathrm{~b}$ ). In those cases, however, the effect is opposite (velocity directed from the yz mid-plane towards the external walls as witnessed by the swap in the color sequence). These regions should therefore produce stretching of the particle structures. In Fig. 11a, it can be noticed, however, that none of them intersects the particle-dense areas.

A close look at the left side of Fig. 11a finally reveals that also in the region of the cubic container occupied by the larger particle structure a compressive effect is at work (two balloon regions pushing fluid towards the central yz plane are also located in that portion of the physical domain). As originally shown in Fig. 10b, however, the shrinkage in size experienced by the left particle structure is relatively contained and the reason of such a behavior resides in the fact that in this case the structure is only marginally influenced by the two regions of fluid where the compressive effect is at work (Fig. 11a). 
Besides their different extension along different spatial directions, another interesting mark distinguishing the two particle structures is the recognizable presence of spiraling filaments, which originate from the smaller one (Fig. 10b) and extend in both the positive and negative $x$ direction.

All these features should be regarded as important differences with respect to earlier situations for which $G s \leq \mathrm{O}\left(10^{3}\right)$. In the light of these arguments, a direct cause-and-effect relationship can therefore be introduced between the observed morphology and asymmetry of the particle attracting regions and the value taken by the Gershuni number.

An indirect (further) confirmation for this interpretation can be yielded by keeping $\gamma$ and $\varpi$ constant and decreasing the Rayleigh number (by making $\beta$ smaller) in such a way that the Gershuni number is decreased by one or two orders of magnitude. This is shown for instance in Fig. 12, which has been obtained for $\gamma=1 \times 10^{8}, \varpi=10^{3}$ and $R a_{\omega}=10^{4}$. A decrease in the Rayleigh number of one order of magnitude leads to a corresponding shrinkage of the Gershuni number, which is scaled accordingly from $\mathrm{O}\left(10^{4}\right)$ to $\mathrm{O}\left(10^{2}\right)$.

In Fig. 12 we have reported again the particle structures (left view) and the related time-averaged velocity field in the yz plane (right view). Cross-comparison with Fig. 9a and 9b can immediately be used to highlight the recovered mirror symmetry of the particle structures and, most importantly, the different behavior taken by the time-averaged velocity field from both qualitative and quantitative standpoints.
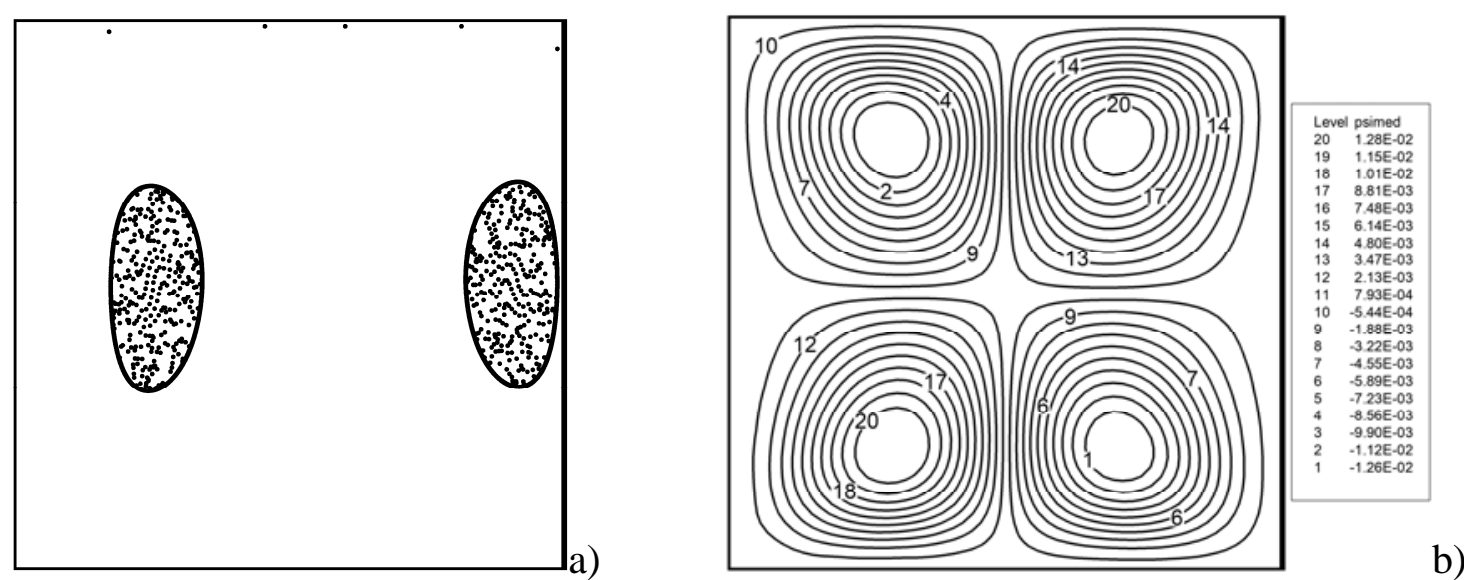

Figure 12: a) snapshot of particle structures in water for $\gamma=1 \times 10^{8}, R a_{\omega}=10^{4}, \varpi=1 \times 10^{3}$ and $\xi=2$ (nondimensional time $\mathrm{t} \cong 10$ ): b) streamlines of the projection of the related time-averaged velocity field in the yz mid-plane $\left(G s \cong 3.05 \times 10^{2}\right)$.

Qualitatively, its structure conforms to the so-called quadrupolar field, namely a set of four counter-rotating rolls symmetrical by reflection with respect to both $z=1 / 2$ and $y=1 / 2$ planes (Fig. 12b), i.e. displaying a perfect center-symmetry ${ }^{45,47,51}$. From a quantitative point of view, the intensity of the time-averaged flow is much smaller (Fig. 12b). Put together, these two observations can be used to justify the morphology of the particle attractors reported in Fig. 12a. It is a natural consequence of the symmetries of the overall problem (there is no effect violating the systems symmetry with respect to the horizontal direction; as explained above, the velocity field shown in 
Fig. $12 \mathrm{~b}$ is a symmetric-symmetric convective mode). As expected, moreover, we could detect no significant particle structure compression effect being effective along the $\mathrm{x}$ direction for the 3D case. To sum up, it can be concluded that at higher values of Gs, the symmetry in the yz plane is broken by the existence of the relatively large vortex created by the coalescence of two of the cells originally contributing to the quadrupolar field (Khallouf et al., ${ }^{77}$; Mialdun et al., ${ }^{45}$; Lappa ${ }^{57}$ ) and by the ability of this spatially extended roll to interfere with the transport of particles owing to its increased strength. Similarly, the tendency of particle structures to detach from the walls delimiting the system along the spanwise direction and be clustered in a more central region (located in a certain neighborhood of the yz mid-plane) is a natural consequence of the time-averaged velocity component along $\mathrm{x}$.
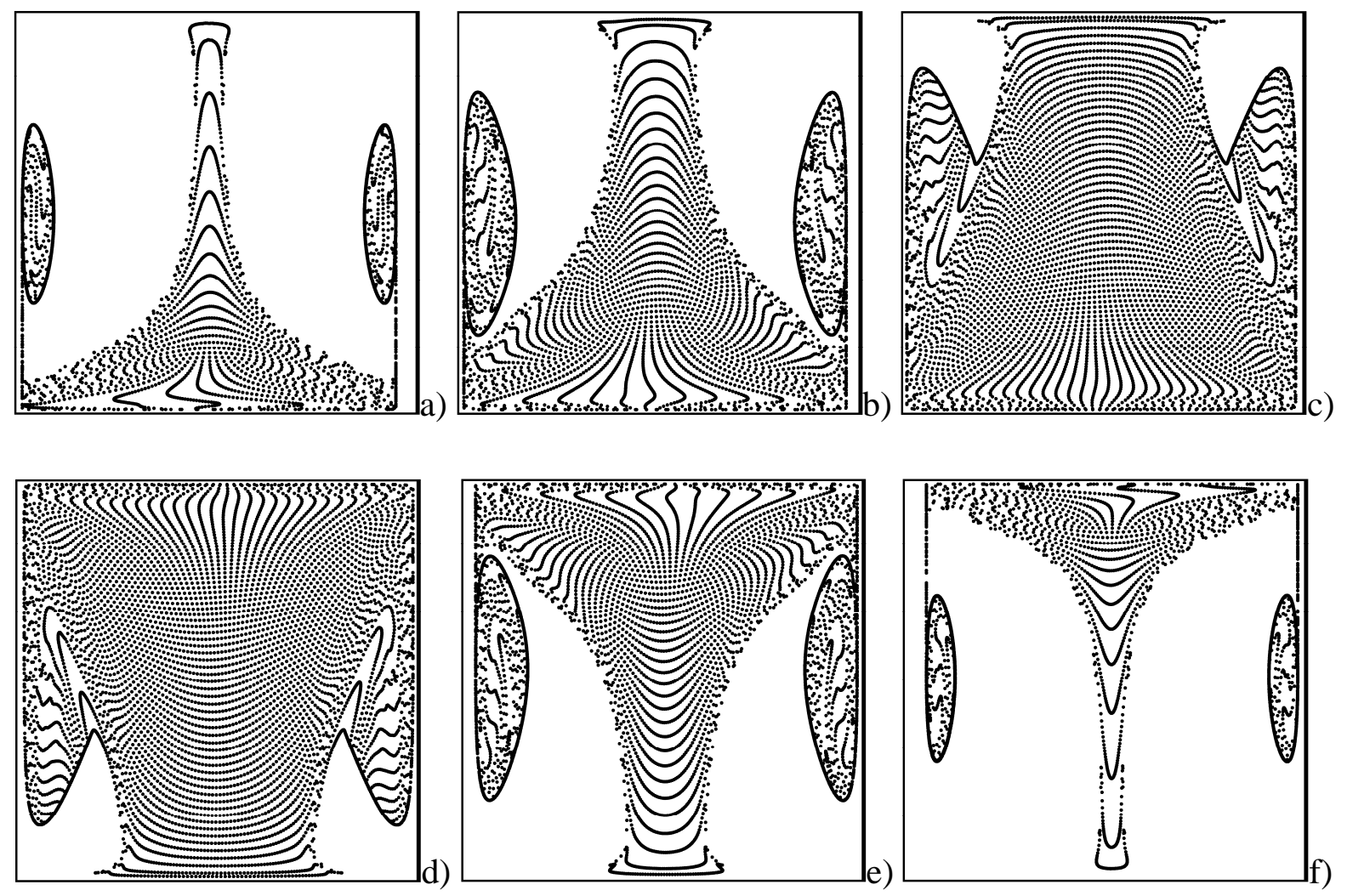

Figure 13: Snapshots of particle structures for $\operatorname{Pr}=15.5, \gamma=2.5 \times 10^{9}, R a_{\omega}=10^{5}, \varpi=3 \times 10^{4}(G s \cong 85)$ and different values of $\xi$ (yz plane, hot side on the top, cold side on the bottom, non-dimensional time $\mathrm{t} \cong 2.5$, 2D computations): a) $\xi=0.1$, b) 0.5 , c) 0.8 , d) 1.2 , e) 1.5 , f) 2.0 .

\subsection{High-Pr-fluid-particles mixtures}

Having completed a sketch of the possible situations for water $(\operatorname{Pr}=6.11)$, we now move to the case of a fluid with larger value of the Prandtl number ( $\operatorname{Pr}=15.5$ corresponding to $1 \mathrm{cSt}$ silicone oil at $25^{\circ} \mathrm{C}$ or ethanol at $33^{\circ} \mathrm{C}$ ).

Beyond the mere motivation to bridge the abovementioned gap (about the type of fluid and the effect of the Prandtl number), we are specifically interested in studying the consequences of high 
values of the Gershuni number. As implied by Eq. (7), indeed, higher values of Gs can be obtained for larger values of Pr using the same angular frequency and Rayleigh number already considered for water. Following the same logical approach undertaken for Sect. 4.1, we consider again situations with an increasing level of complexity as the discussion progresses.

Along these lines, Fig. 13 reports in an ordered way the structures emerging in the cavity for $\gamma=2.5 \times 10^{9}, R a_{\omega}=10^{5}$, and $\varpi=3 \times 10^{4}$ and various density ratios $\xi$. It can be seen that for such a set of parameters (mathematically equivalent to a value of the Gershuni number relatively small, i.e. $G s \cong 85$ ), the attractors recover a perfectly symmetrical shape with respect to the plane $y=0$.

The corresponding distribution of sizes as a function of $\xi$ can be gathered from Fig. 14. As the reader will easily realize by cross comparison with the earlier results obtained for water and $G s \cong 305$, the trends are relatively similar.
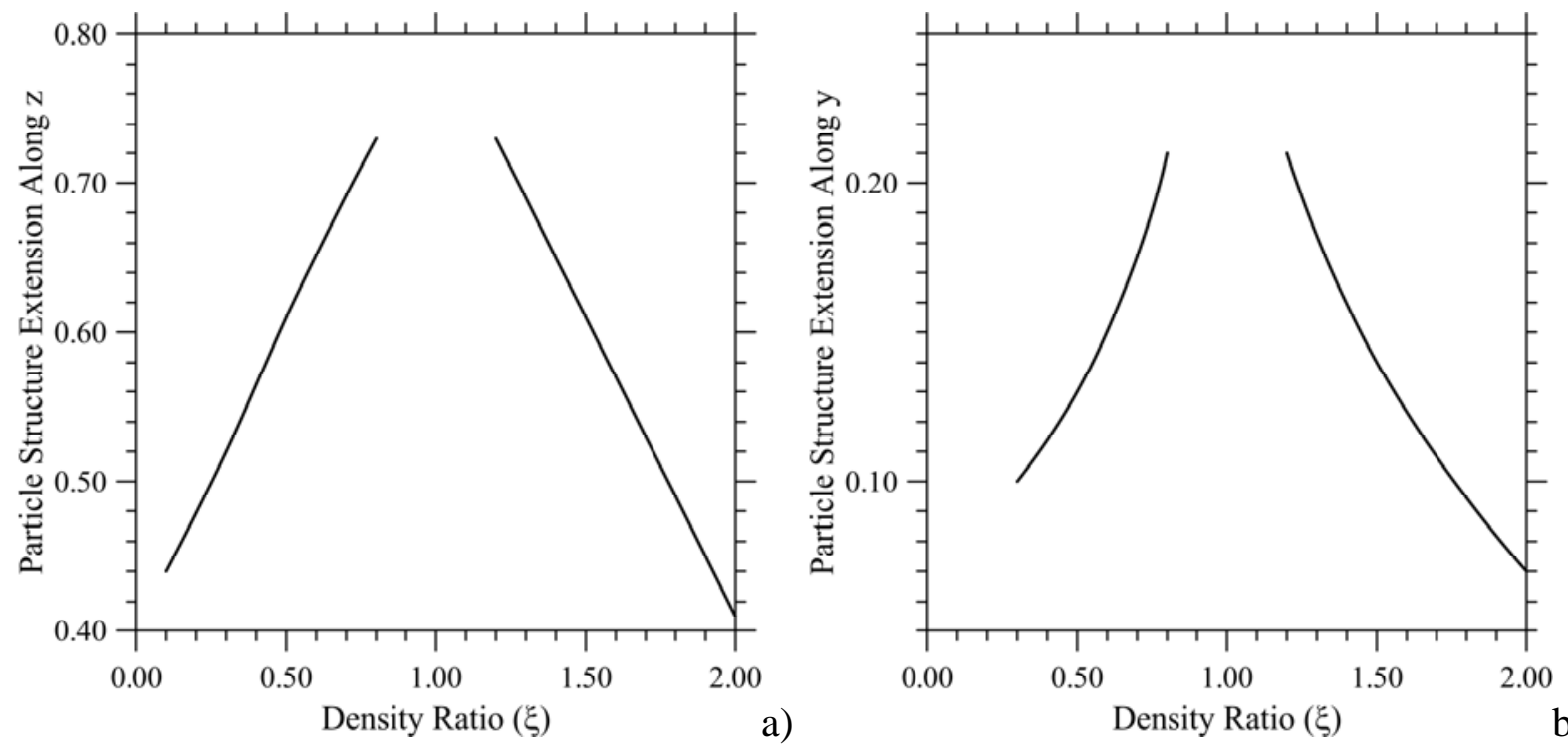

Figure 14: Spatial extension of particle structures along the $\mathrm{z}(\mathrm{a})$ and $\mathrm{y}(\mathrm{b})$ axes as a function of the density ratio for $\gamma=2.5 \times 10^{9}, R a_{\omega}=10^{5}, \varpi=3 \times 10^{4}(G s \cong 86)(\operatorname{Pr}=15.5)$.

\subsubsection{Symmetry breaking phenomena}

An increase in $R a_{\omega}$ for fixed $\varpi$, however, leads again to an evident symmetry breaking in the morphology of the particle structures (Fig. 15).

These dynamics are consistent with the related value of $G s(\cong 8600)$, which implies a relatively strong magnitude of the time-averaged velocity (which, as illustrated before, is able to influence significantly particle motion and interfere with the delicate mechanisms leading particles to be constrained in specific regions of the physical domain). As evident in Fig. 15, the patterns formed by the particles are so distorted that proper characterization of their morphology requires a separate description of the left and right structures and related size scaling laws. These can be inferred from Fig. 16, where different symbols (closed circles and triangles) have been used to distinguish the data relating to the two opposing particle structures. Interestingly, these figures show that the 
difference in terms of size along z (Fig. 16a) and along y (Fig. 16b) of the two structures is reduced as the quantity $|\xi-1|$ is increased in magnitude (i.e. for relatively small or high values of $\xi$; similar conclusions could directly be drawn by inspecting the patterning behavior illustrated in Fig. 15).
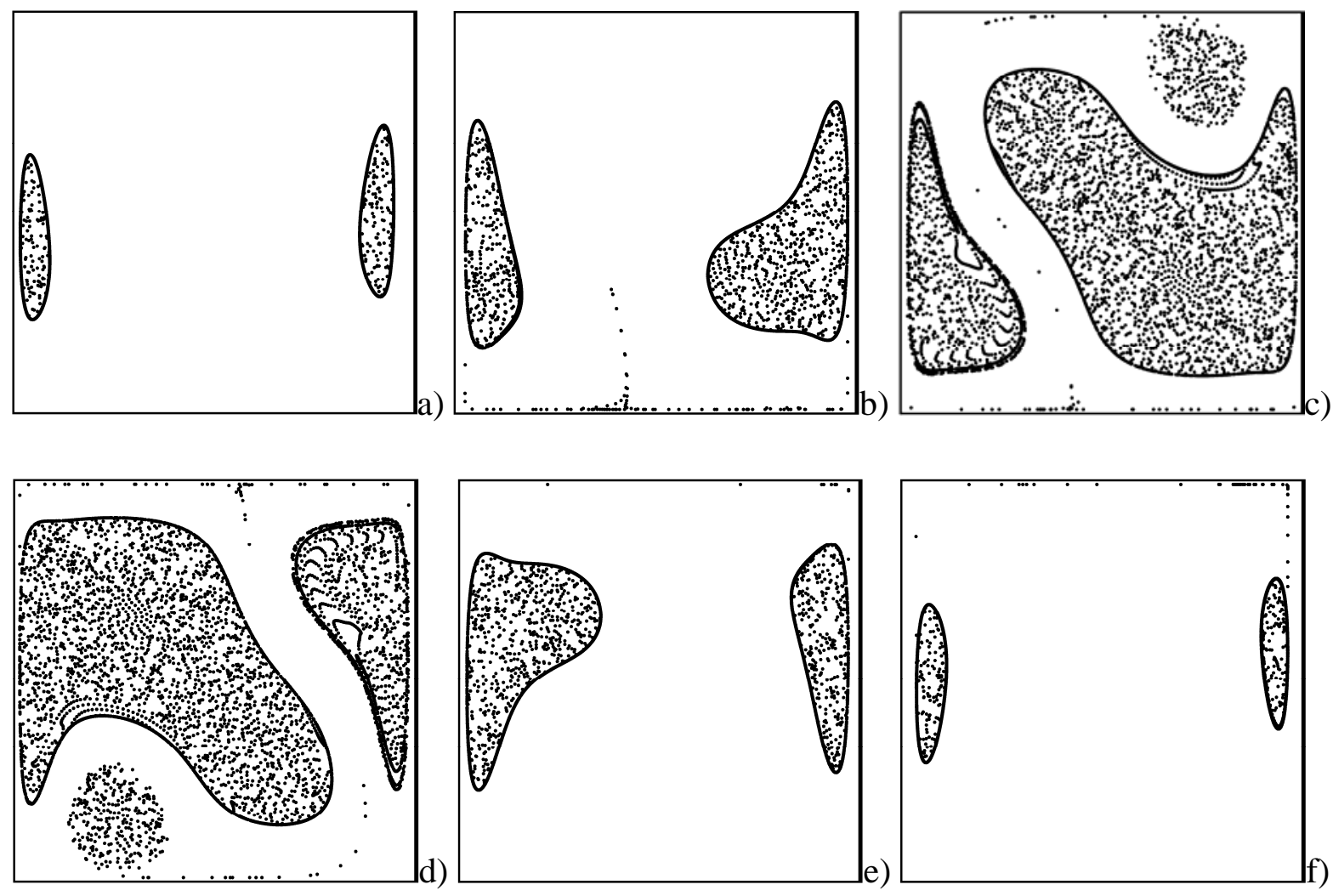

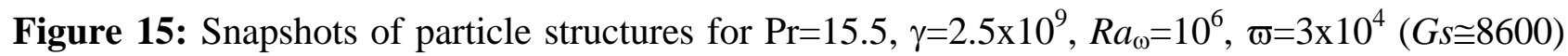
and different values of $\xi$ (yz plane, hot side on the top, cold side on the bottom, non-dimensional time $\simeq 2.5$ ): a) $\xi=0.1$, b) 0.5 , c) 0.8 , d) 1.2 , e) 1.5 , f) 2.0 .

\subsubsection{Influence of the Rayleigh number}

Additional insights follow naturally from a comparison of Figs. 16 and 14. As a fleeting glimpse into these figures would confirm, indeed, for given values of $\gamma$ and $\varpi$, the size of the structures along the direction of the temperature gradient ( $\mathrm{z}$ axis) can be assumed to be weakly dependent on the value taken by the Rayleigh number. 

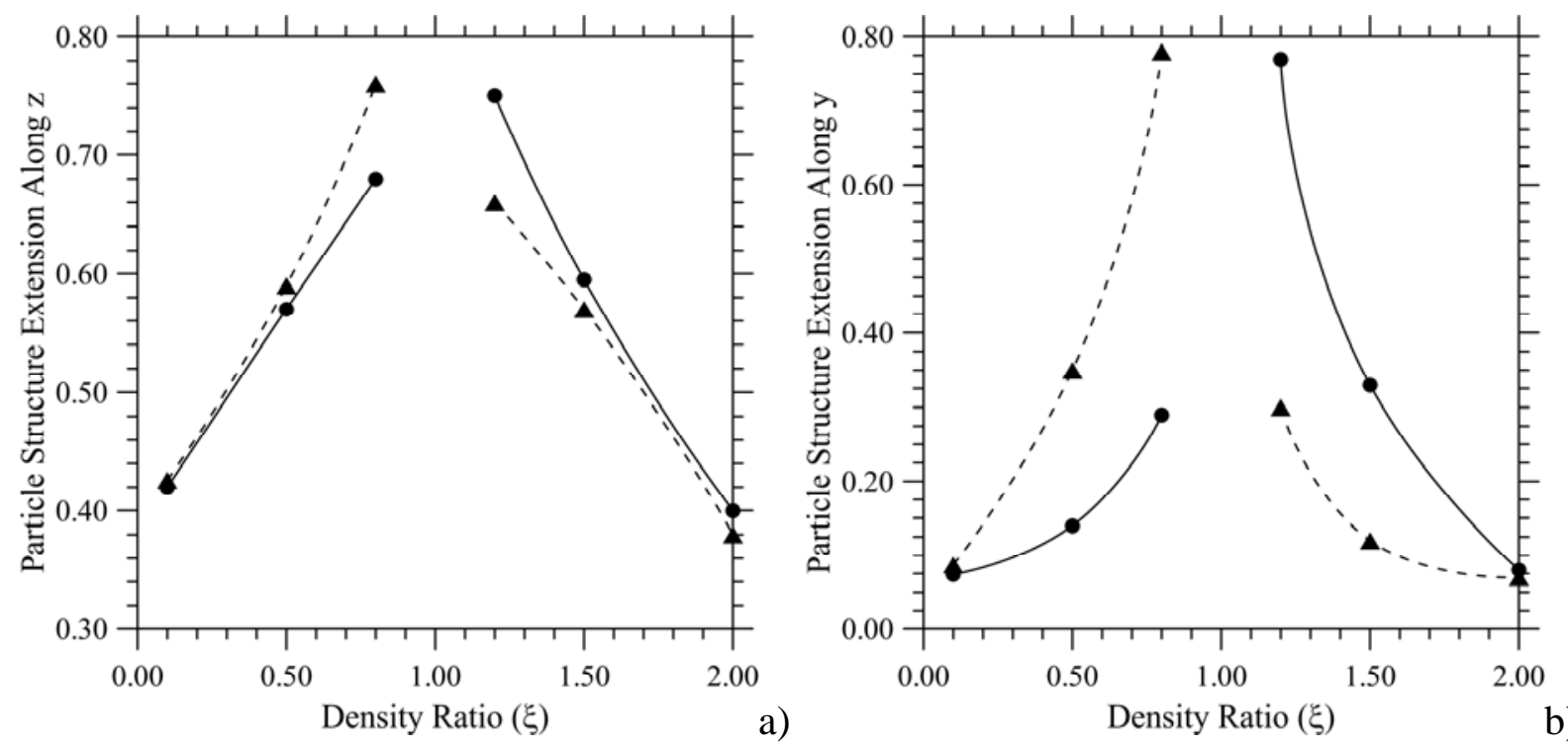

Figure 16: Spatial extension of particle structures along the $\mathrm{z}(\mathrm{a})$ and $\mathrm{y}(\mathrm{b})$ axes as a function of the density ratio for the same conditions considered in Fig. 15: closed circles (solid line) - left structure, triangles (dashed line) - right structure.

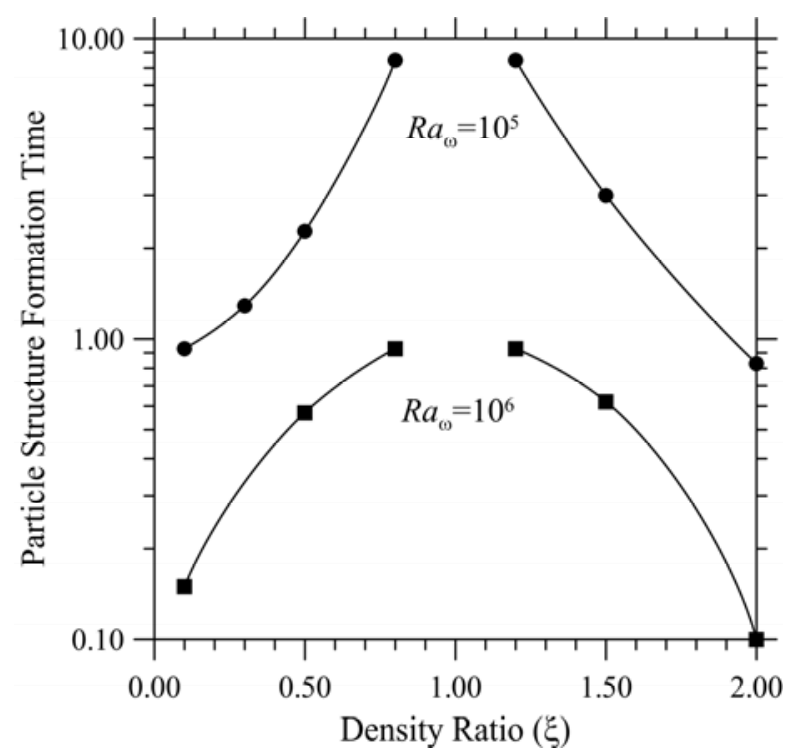

Figure 17: Particle structure formation time as a function of the density ratio for $\gamma=2.5 \times 10^{9}$, $\varpi=3 \times 10^{4}$ and two values of $R a_{\omega}(\operatorname{Pr}=15.5)$.

A separate discussion, however, is needed for the impact of this parameter on the formation time. As revealed by Fig. 17, the influence of $R a_{\omega}$ on the temporal evolution of these phenomena is remarkable. On increasing $R a_{\omega}$ by one order of magnitude, an equivalent variation is induced in the formation time (decreasing as a function of $R a_{\omega}$ ). This figure is also instrumental in showing that the asymmetry of the two branches (evident by comparison of the data for $\xi=0.5$ and 1.5 for instance) is enhanced when the Rayleigh number takes higher values. This trend can directly be ascribed to an increase in the relative importance of the influence of drag and the added mass (with 
respect to density mismatch effects) when the strength of thermovibrational convection grows (as a result of an increase in $R a_{\omega}$ ).

The strong dependence of the structure size on the value of the acceleration amplitude $(\gamma)$ is obviously retained when water is replaced with a higher-Pr liquid (Figs. 18-20). Snapshots witnessing this dependence for $R a_{\omega}=10^{5}, \xi=2$ have been grouped in Fig. 18 and Fig. 20 for $\varpi=10^{4}$ and $\varpi=3 \times 10^{4}$, respectively (for $\varpi=10^{4}$, the reader is also referred to Fig. 19 for the corresponding 3D simulations). As expected, a variation of $\gamma$ at fixed $R a_{\omega}$ and $\varpi$ can cause a significant alteration of the attractor size. Interestingly, due to the interaction of the particles with the time-averaged velocity field and its peculiar topological properties, the unstable reservoir of particles, which for smaller values of $G s$ is generally located in the intermediate region between the two main attractors, now occupies one of the cavity corners (the lack of a well-defined line separating it from the clear fluid outside can still be used as a distinguishing criterion). Figure 19 finally confirms that for ( $G s \cong \mathrm{O}\left(10^{4}\right)$ or larger) the morphological changes are not limited to the emergence of a visible asymmetry in the shape of the two stable accumulation loci in the yz plane. The less spatially extended particle structure is also compressed along the spanwise direction (x) and displays recognizable helicoidal filaments, with turns that become progressively more spaced as they approach the $\mathrm{x}=-0.5$ and $\mathrm{x}=0.5$ sidewalls (Fig. 19b).
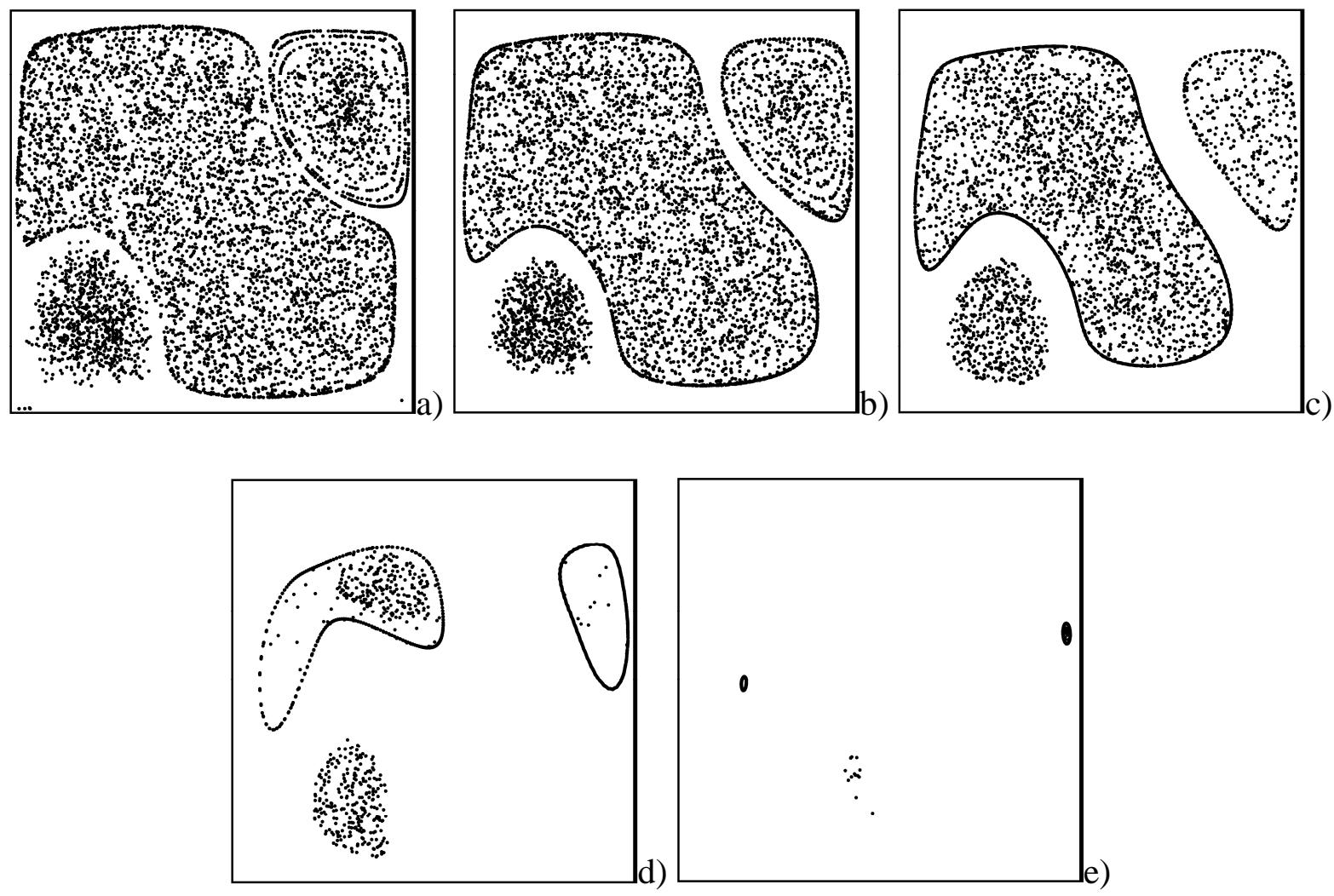

Figure 18: Snapshots of particle structures for $\operatorname{Pr}=15.5, R a_{\omega}=10^{6}, \varpi=10^{4}\left(G s \cong 7.7 \times 10^{4}\right), \xi=2$ and different values of the acceleration amplitude $\gamma$ (yz plane, hot side on the top, cold side on the bottom, non-dimensional time $t \simeq 2.5$ ): a) $\gamma=10^{8}$, b) $2.5 \times 10^{8}$, c) $\gamma=5 \times 10^{8}$, d) $\gamma=1 \times 10^{9}$, e) $\gamma=2.5 \times 10^{9}$. 

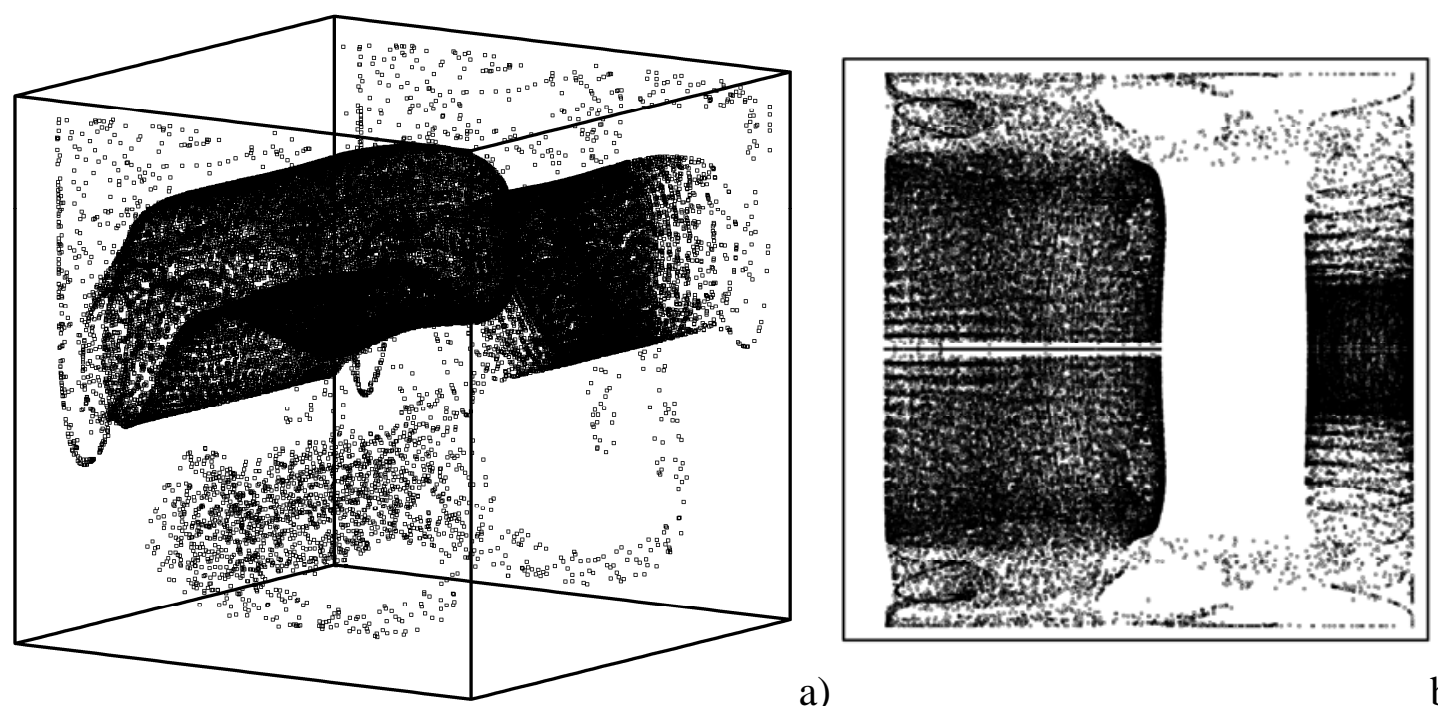

a)

b)

Figure 19: Snapshot of particle structures for $\operatorname{Pr}=15.5, \gamma=1 \times 10^{9}, R a_{\omega}=10^{6}, \varpi=1 \times 10^{4}\left(G s \cong 7.7 \times 10^{4}\right)$ and $\xi=2$ (non-dimensional time $\mathrm{t}=2.5$ ): a) $3 \mathrm{D}$ view, b) top view (along $\mathrm{z}$ )
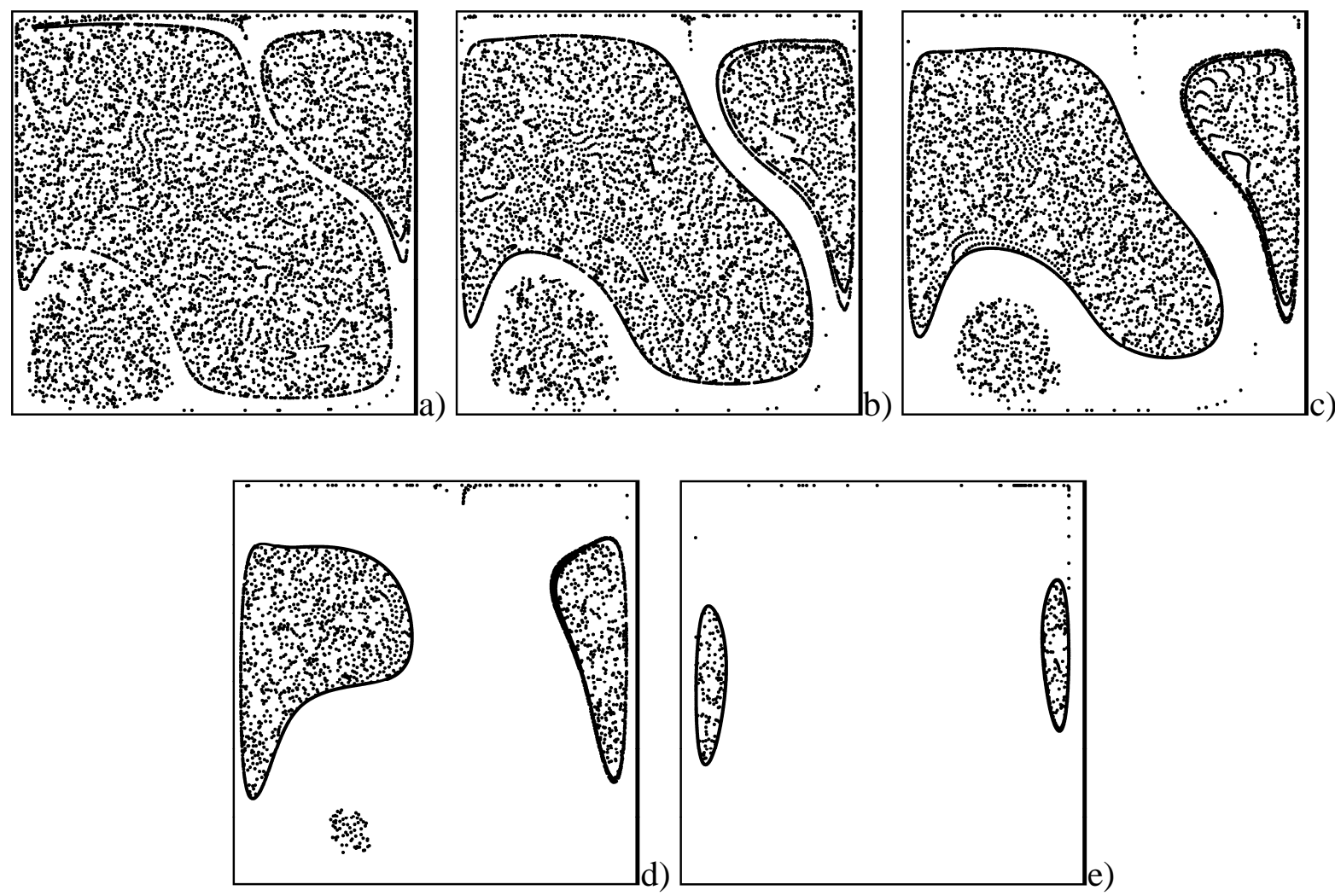

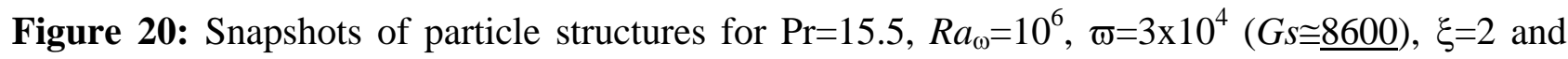
different values of the acceleration amplitude $\gamma$ (yz plane, hot side on the top, cold side on the bottom, non-dimensional time $t \simeq 2.5$ ): a) $\gamma=10^{8}$, b) $2.5 \times 10^{8}$, c) $\gamma=5 \times 10^{8}$, d) $\gamma=1 \times 10^{9}$, e) $\gamma=2.5 \times 10^{9}$. 


\subsubsection{Frequency effects}

By taking a close look at Figs. 18 and 20 it can also be discerned that an increase in $\varpi$ while maintaining unchanged $\gamma$ and $R a_{\omega}$ can have a remarkable multi-fold impact. First of all, it causes a decrease in the value of Gs. Accordingly, the degree of distortion of the particle patterns tends to be mitigated. At the same time, the structures become larger, which is a relatively counterintuitive finding if one considers that higher values of $\varpi$ would indicate a larger amount of energy being injected in the system.

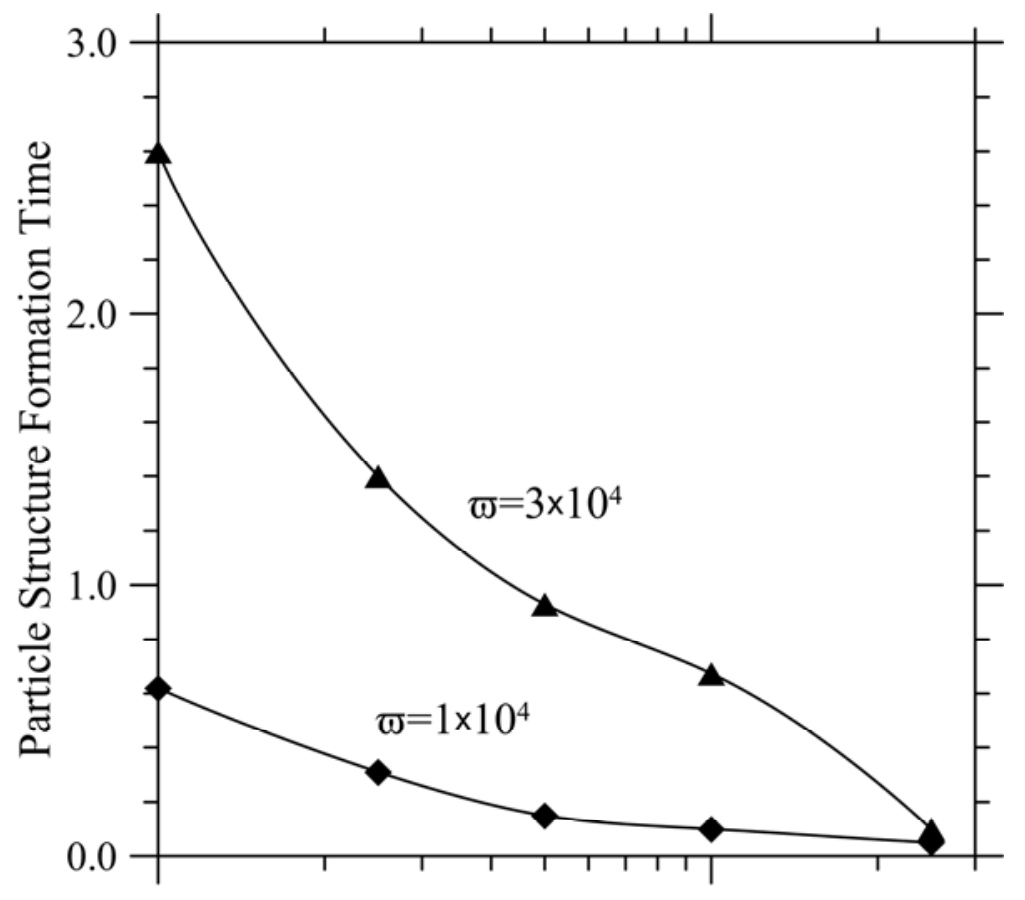

$1 \mathrm{E}+008$

$1 \mathrm{E}+009$

Acceleration Amplitude $(\gamma)$

Figure 21: Particle structure formation time as a function of the acceleration amplitude for $R a_{\omega}=$ $10^{6}, \xi=2$ and two different values of $\varpi(\operatorname{Pr}=15.5)$.

The most striking (an unexpected) effect produced by an increase in $\varpi$, however, is the longer time taken by structures for their formation. This trend has been summarized in Fig. 21.

The formation time for $\varpi=3 \times 10^{4}$ always lies above that for $\varpi=10^{4}$. Interestingly, however, the delay represented by the vertical distance between the two diagrams tends to be reduced as $\gamma$ becomes higher (until it takes almost negligible values when $\gamma$ exceeds $2 \times 10^{9}$ ).

In order to explore further this relationship and filter out the distortions induced by time-averaged effects, we have performed other simulations maintaining fixed $\xi, \gamma$ and $R a_{\omega}\left(10^{5}\right)$ and varying parametrically $\varpi$ is such a way to keep $G$ s smaller than $\mathrm{O}\left(10^{4}\right)$. The outcomes of such simulations are summarized in Fig. 22 for $\varpi$ spanning the interval $5 \times 10^{3} \leq \varpi \leq 3 \times 10^{4}$.

The related characteristic dimensions of the structures and formation times have been collected in Fig. 23, where it can be seen that the dependence of the formation time on $\varpi$ is essentially parabolic. 

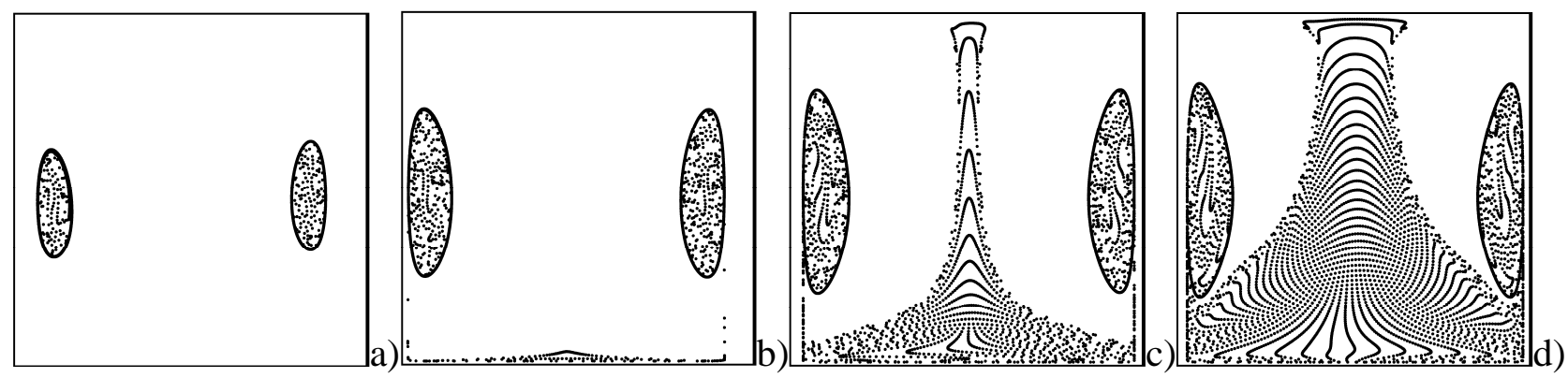

Figure 22: Snapshots of particle structures for $\operatorname{Pr}=15.5, \gamma=2.5 \times 10^{9}, R a_{\omega}=10^{5}, \xi=0.5$ as a function of the angular frequency of vibrations $\varpi$ (yz plane, hot side on the top, cold side on the bottom, nondimensional time $t \cong 2.5$, 2D computations): a) $\varpi=5 \times 10^{3}$, b) $\varpi=1 \times 10^{4}$, c) $\varpi=2 \times 10^{4}$, d) $\varpi=3 \times 10^{4}$.
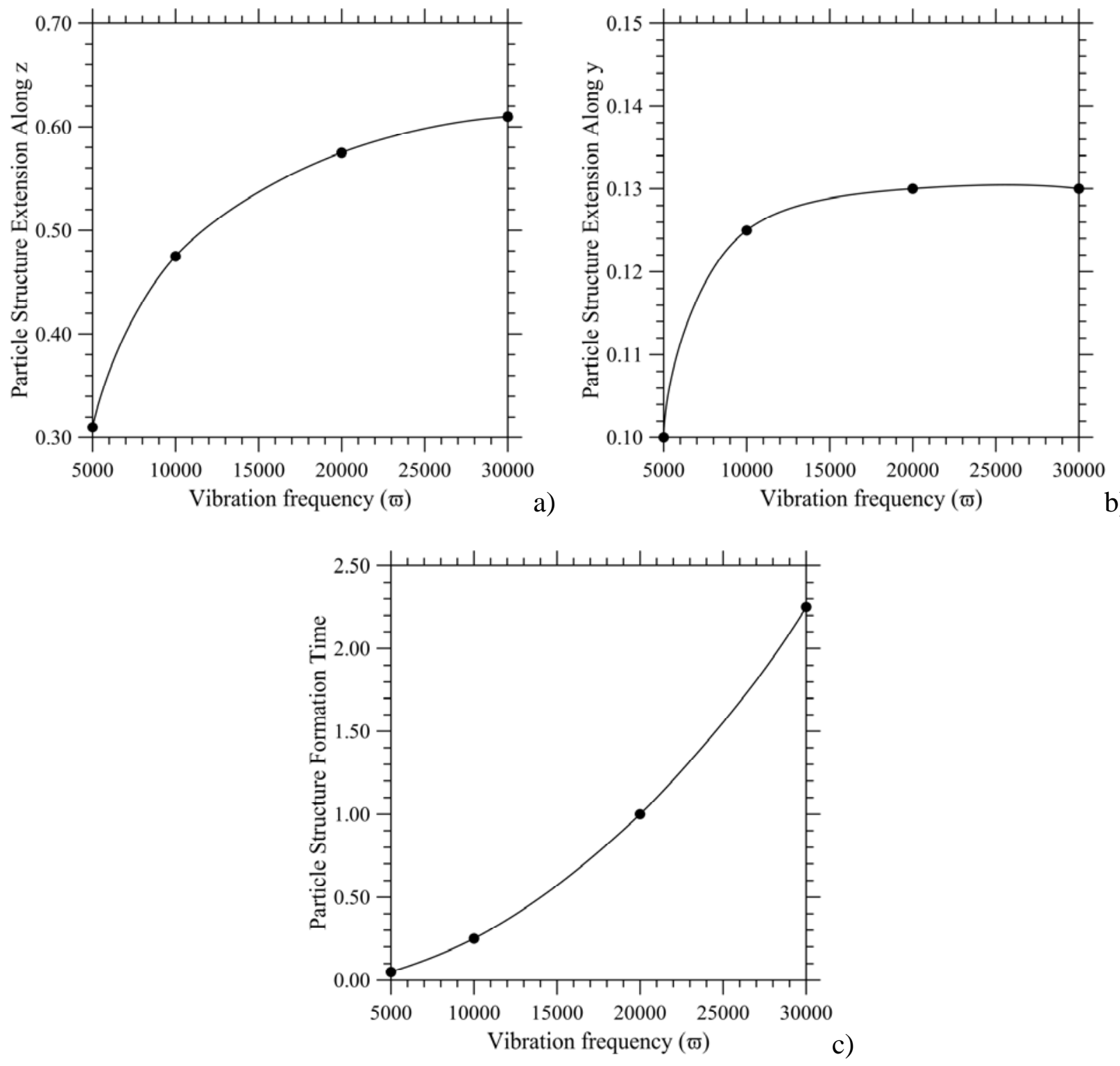

Figure 23: Spatial extension of particle structures ((a) and (b)) and related formation time (c) as a function of the angular frequency of vibrations for $\gamma=2.5 \times 10^{9}, R a_{\omega}=10^{5}$ and $\xi=0.5(\operatorname{Pr}=15.5)$. 


\subsection{A generalized correlation for the structure formation time}

In this last section, we finally devote some effort to present a general correlation (analytical formula) for the particle structure formation time $\left(t_{\text {form }}\right)$ able to account for all the functional relationships discussed in the preceding sections and provide reliable quantitative estimates.

Remarkably, using all the available data about the variations of $t_{\text {form }}$ with $\varpi, \xi, \gamma$, and $R a_{\omega}$, we could successfully condense all such dependences into a single analytical expression, i.e.:

$$
t_{\text {form }}=C \frac{1}{|\xi-1|^{3 / 2}} \frac{\varpi^{2}}{R a_{\omega} \gamma}
$$

with the value of the constant $C$ being independent from the Prandtl number $\left(C=2.2 \times 10^{5} \cong S t^{-1}\right)$. In agreement with all the trends presented in Figs. 4, 6c, 8c, 17, 21 and 23c, this formula shows a quadratic relationship between the formation time and the vibrations angular frequency, while direct inverse proportionality can be seen with respect to the Rayleigh number and the non-dimensional acceleration amplitude (doubling one of these parameters would reduce $t_{\text {form }}$ by half). The link with the density ratio is slightly more complex as in this case a corrective exponent (3/2) must be considered for the factor $|\xi-1|$ present at the denominator of the formula.

The compactness of this formula will prove very useful for the definition of "ad hoc" experiments to be executed on orbiting (microgravity) platforms.

\section{Conclusions}

Within the vast array of problems encompassed by two-phase flows, the main focus of the present work was an analysis of the peculiar phenomena driven by the interplay of thermovibrational flow and solid particles dispersed in a non iso-dense liquid. The recent resurgence in interest in this specific subject has been driven by the discovery of a new category of particle attractors. These accumulation loci always manifest in the physical space as a couple of disjoint particle structures, however, their morphology and related degrees of spatial symmetry can be controlled by changing the direction of vibrations (Lappa ${ }^{37,54-55}$ ). The present work has been structured around the main objective of investigating further the evolution of these structures when, as a new factor contributing to their variety, spontaneous symmetry breaking phenomena start to affect the dynamics of the carrier flow.

For these reasons, all the attention has been concentrated on a single archetypal setting, i.e. the case in which vibrations are perfectly perpendicular to the walls of the container and the imposed temperature difference. By varying the independent parameters governing the considered dynamics, we have shown that the mirror symmetry of the two tubular particle accumulation surfaces (reported in earlier studies on this subject) can be broken when the time-averaged flow becomes significant. 
The related pattern in planes containing vibrations and the applied temperature difference, initially displaying a central symmetry (the so-called quadrupolar velocity field), quickly undergoes bifurcation to a no-longer symmetric state (single-vortex regime), which also features a component in the spanwise direction. This roll can transfer particles from the area of influence of one attractor to that of the other thereby causing an evident imbalance in the size of the two emerging particle structures. In turn, the component of time-averaged velocity along the third direction can exert a compressive action on the particle structure having smaller transverse size causing shrinkage of its extension also along the spanwise (x) direction.

As a result of all these effects, the location, size and shape of the areas where particles tend to be clustered can be significantly affected, which is a previously undiscovered phenomenon.

As a collateral outcome of the above-mentioned parametric investigation, the existence of an 'unstable' particle attracting region has also been revealed, i.e. a specific portion of the physical space where particles tend to be temporarily collected before they are finally trapped within the two main (stable) attractors. Also this attractor can 'feel' the presence of a time-averaged component of fluid velocity. As a result, it can be displaced from the center of the container (where it is located for relatively small values of the Gershuni number) to one of its corners.

Despite the significant computational cost being associated with the numerical approach used to investigate these dynamics (based on the solution of the Navier-Stokes equations in their complete, time-dependent and non-linear form, though partially mitigated by the possibility to conduct part of the explorative simulations in a two-dimensional framework), we have carefully identified the various functional dependences linking the temporal and spatial properties of the particle structures to the relevant influential (independent) parameters. Some final effort has been provided to turn all the data obtained into a generalized analytical formula for the structure formation time, which will prove very useful for the optimization of a set of experiments currently planned for execution onboard the International Space Station.

\section{Acknowledgments}

This work has been supported by the UK Space Agency (grant ST/S006354/1) in the framework of the T-PAOLA (Particle-Vibration) project.

\section{Data Availability}

The data that support the findings will be available in a repository.

\section{References}

[01] Schwabe D., Mizev A.I., Udhayasankar M. and Tanaka S., (2007), Formation of dynamic particle accumulation structures in oscillatory thermocapillary flow in liquid bridges, Phys. Fluids, 19(7), 072102. 
[02] Pushkin D., Melnikov D., Shevtsova V., (2011), Ordering of Small Particles in OneDimensional Coherent Structures by Time-Periodic Flows, Phys. Rev. Lett., 106, 234501.

[03] Melnikov D.E., Pushkin D.O., Shevtsova V.M., (2013), Synchronization of finite-size particles by a traveling wave in a cylindrical flow, Phys Fluids, 25 (9), 092108.

[04] Gotoda M., Melnikov D. E., Ueno I., and Shevtsova V., (2016), Experimental study on dynamics of coherent structures formed by inertial solid, particles in three-dimensional periodic flows, Chaos, 26, 073106 (11 pages).

[05] Parthasarathy R. N. and Faeth G. M., (1990), Turbulent dispersion of particles in self-generated homogeneous turbulence, J. Fluid Mech., 220, 515-537.

[06] Ehara K., Hagwood C., Coakley K.J., (1996), Novel method to classify aerosol particles according to their mass-to-charge ratio-Aerosol particle mass analyzer, J. Aerosol Sci. 27, 217-234. [07] Lappa M., (2019), “On the Nature of Fluid-dynamics”, Chapter 1 (pp. 1-64) in Understanding the Nature of Science, Patrick Lindholm Editor, Nova Science Publishers Inc., Series: Science, Evolution and Creationism, BISAC: SCI034000, ISBN: 978-1-53616-016-1, https://novapublishers.com/shop/understanding-the-nature-of-science/

[08] Bracco A., Chavanis P. H., Provenzale A., Spiegel E. A., (1999), Particle aggregation in a turbulent Keplerian flow, Phys. Fluids, 11( 8), 2280-2287.

[09] Lappa M., (2016), On the nature, formation and diversity of particulate coherent structures in Microgravity Conditions and their relevance to materials science and problems of Astrophysical interest, Geophysical and Astrophysical Fluid Dynamics, 110(4), 348-386.

[10] Haszpra T. and Tél T., (2011), Volcanic ash in the free atmosphere: A dynamical systems approach, Journal of Physics: Conference Series 333, 012008 (12 pages).

[11] Srivastava V.P., (1995), Particle-fluid suspension model of blood flow through stenotic vessels with applications, International Journal of Bio-Medical Computing, 38(2), 141-154.

[12] Saghir M. Z. and Mohamed A., (2018), Effectiveness in incorporating Brownian and thermophoresis effects in modelling convective flow of water- $\mathrm{Al}_{2} \mathrm{O}_{3}$ nanoparticles, Int. J. Numer. Methods Heat Fluid Flow 28(1), 47-63.

[13] Aquaro D., (2006), Erosion due to the impact of solid particles of materials resistant at high temperature, Meccanica, 41, 539-551.

[14] Dasgupta S., Jackson R., Sundaresan S., (1998), Gas-particle flow in vertical pipes with high mass loading of particles, Powder Technology, 96, 6-23.

[15] Rao C.S.R, (1977), Fluidized-Bed Combustion Technology-A Review, Combustion Science and Technology, 16(3-6), 215-227.

[16] Zhao J. Z., Ratke L. and Feuerbacher B., (1998), Microstructure evolution of immiscible alloys during cooling through the miscibility gap, Modelling Simul. Mater. Sci. Eng., 6, 123-139.

[17] Balboa-Usabiaga F., Delgado-Buscalioni R., (2011), Particle hydrodynamics: From molecular to colloidal fluids, Particle-Based Methods II - Fundamentals and Applications, Springer Nature, pp. 152-163.

[18] Eaton J.K. and Fessler J.R., (1994), Preferential concentration of particles by turbulence, Int. J. Multiphase Flow, 20, 169-209.

[19] Jayaram R., Jie Y., Zhao L., Andersson H.I., (2020), Clustering of inertial spheres in evolving Taylor-Green vortex flow featured, Phys. Fluids, 32, 043306.

[20] Yuan W., Zhao L., Andersson H. I., and Deng J., (2018), Three-dimensional Voronoï analysis of preferential concentration of spheroidal particles in wall turbulence, Phys. Fluids 30, 063304.

[21] Luo K., Wang Z., Li D., Tan J. and Fan J., (2017), Fully resolved simulations of turbulence modulation by high-inertia particles in an isotropic turbulent flow, Phys. Fluids, 29, 113301.

[22] Haller G. and Sapsis T., (2008), Where do inertial particles go in fluid flows?, Physica D 237(5), 573-583.

[23] Love S.G. and Pettit D.R., (2004), Fast repeteable clumping of solid particles in microgravity, Lunar and Planetary Science, XXXV. 
[24] Tabakova S.S. and Zapruanov Z.D., (1982), On the hydrodynamic interaction of two spheres oscillating in a viscous fluid. I. Axisymmetrical case. J. Appl. Math. Phys. (ZAMP), 33, 344-357 II. Three dimensional case. J. Appl. Math. Phys. (ZAMP), 33, 487-502.

[25] Wunenburger R., Carrier V., and Garrabos Y., (2002), Periodic order induced by horizontal vibrations in a two-dimensional assembly of heavy beads in water, Phys. Fluids, 14(7), 2350-2359. [26] Thomas C.C. and Gollub J.P., (2004), Structures and chaotic fluctuations of granular clusters in a vibrated fluid layer, Phys. Rev. E 70, 061305.

[27] Ivanova A.A., Kozlov V.G. and Kuzaev A.F., (2005), Vibrational lift force acting on a body in a fluid near a solid surface. Dokladi RAN, vol. 402 (4), pp. 1-4 (Translated: Doklady Physics, vol. 50 (6), pp. 311-314).

[28] Kozlov V.G., Ivanova A.A. and Evesque P., (2006), Block stratification of sedimenting granular matter in a vessel due to vertical vibration, Fluid Dyn. Mater. Process., 2(3), 203-210.

[29] Lyubimov D.V., Lyubimova T.P., Straube A.V., (2005), Accumulation of Solid Particles in Convective Flows, Microgravity Sci. Technol., XVI - I, 210-214.

[30] Melnikov D., Pushkin D., and Shevtsova V., (2011) Accumulation of particles in timedependent thermocapillary flow in a liquid bridge. Modeling of experiments, Eur. Phys. J. Special Topics, 192, 29-39.

[31] Melnikov, D.E., Watanabe, T., Matsugase, T., Ueno, I., Shevtsova, V., (2014), Experimental study on formation of particle accumulation structures by a thermocapillary flow in a deformable liquid column, Microgravity Sci. Technol. 26, 365-374.

[32] Melnikov D.E. and Shevtsova V., (2017), Different types of Lagrangian coherent structures formed by solid particles in three-dimensional time-periodic flows, European Physical Journal: Special Topics, 226(6), 1239-1251.

[33] Lappa M., (2013), Assessment of the role of axial vorticity in the formation of Particle Accumulation Structures (PAS) in supercritical Marangoni and hybrid thermocapillary-rotationdriven flows, Phys. Fluids, 25(1) 012101 (11 pages).

[34] Lappa M., (2013), On the Existence and Multiplicity of One-dimensional Solid Particle Attractors in Time-dependent Rayleigh-Bénard Convection, Chaos, 23(1), 013105 (9 pages).

[35] Lappa M., (2013), On the variety of particle accumulation structures under the effect of gjitters, J. Fluid Mech., 726, 160-195.

[36] Lappa M., (2014), Stationary Solid Particle Attractors in Standing Waves, Phys. Fluids, 26(1), 013305 (12 pages).

[37] Lappa M., (2014), The patterning behavior and accumulation of spherical particles in a vibrated non-isothermal liquid, Phys. Fluids, 26(9), 093301.

[38] Lappa M., (2019), Towards new contact-less techniques for the control of inertial particles dispersed in a fluid, 12th International Conference on Thermal Engineering, February 23-26, 2019, PDPU, Gandhinagar, Gujarat, India.

[39] Tanaka S., Kawamura H., Ueno I., and Schwabe D., (2006), Flow structure and dynamic particle accumulation in thermocapillary convection in a liquid bridge, Phys. Fluids, 18, 067103.

[40] Ueno I., Abe Y., Noguchi K., and Kawamura H., (2008), Dynamic particle accumulation structure (PAS) in half-zone liquid bridge - Reconstruction of particle motion by 3-D PTV, Adv. Space Res., 41(12), 2145-2149.

[41] Schwabe D. and Mizev A. I., (2011), Particles of different density in thermocapillary liquid bridges under the action of travelling and standing hydrothermal waves, Eur. Phys. J. Spec. Top. 192, 13-27.

[42] Gotoda M., Sano T., Kaneko T., and Ueno I., (2015), Evaluation of existence region and formation time of particle accumulation structure (PAS) in half-zone liquid bridge, Eur. Phys. J. Special Topics, 224, 299.

[43] Toyama A., Gotoda M., Kaneko T., Ueno I., (2017), Existence Conditions and Formation Process of Second Type of Spiral Loop Particle Accumulation Structure (SL-2 PAS) in Half-zone Liquid Bridge, Microgravity Sci. Technol., 29:263-274. 
[44] Gershuni G.Z. and Lyubimov D.V.,(1998), Thermal Vibrational Convection Wiley, England. [45] Mialdun A., Ryzhkov I.I., Melnikov D.E., and Shevtsova V., (2008), Experimental Evidence of Thermal Vibrational Convection in a Nonuniformly Heated Fluid in a Reduced Gravity Environment, Phys. Rev. Lett., 101, 084501.

[46] Lyubimova T.P., Perminov A.V., Kazimardanov M.G. (2019), Stability of quasi-equilibrium states and supercritical regimes of thermal vibrational convection of a Williamson fluid in zero gravity conditions, Int. J. Heat Mass Transf., 129, 406-414.

[47] Bouarab S., Mokhtari F., Kaddeche S., Henry D., Botton V., and Medelfef1 A., (2019), Theoretical and numerical study on high frequency vibrational convection: Influence of the vibration direction on the flow structure, Phys. Fluids, 31(4), 043605.

[48] Shevtsova V., Lyubimova T., Saghir Z., Melnikov D., Gaponenko Y., Sechenyh V., Legros J. C., and Mialdun A., (2011), IVIDIL: On-board g-jitters and diffusion controlled phenomena, $J$. Phys.: Conf. Ser. 327, 012031.

[49] Shevtsova V., Mialdun A., Melnikov D., Ryzhkov I., Gaponenko Y., Saghir Z., Lyubimova T., and Legros J. C., (2011), The IVIDIL experiment onboard the ISS: Thermodiffusion in the presence of controlled vibrations, Compt. Rend. Mécaniq. 339(5), 310-317.

[50] Maryshev B., Lyubimova T., and Lyubimov D., (2013), Two-dimensional thermal convection in porous enclosure subjected to the horizontal seepage and gravity modulation, Phys. Fluids 25, 084105.

[51] Lappa M., (2016), Control of convection patterning and intensity in shallow cavities by harmonic vibrations, Microgravity Science \& Technology, 28(1), 29-39.

[52] Vorobev A. and Lyubimova T., (2019), Vibrational convection in a heterogeneous binary mixture. Part I. Time-averaged equations, J. Fluid Mech., 870, 543-562.

[53] Lappa M., (2016), Numerical study into the morphology and formation mechanisms of threedimensional particle structures in vibrated cylindrical cavities with various heating conditions, Physical Review Fluids, 1(6), 064203.

[54] Lappa M., (2017), On the multiplicity and symmetry of particle attractors in confined nonisothermal fluids subjected to inclined vibrations, Int. J. Multiphase Flow, 93, 71-83.

[55] Lappa M., (2019), On the formation and morphology of coherent particulate structures in nonisothermal enclosures subjected to rotating g-jitters, Phys. Fluids, 31(7), 073303 (11 pages).

[56] Lappa M., (2019), Time reversibility and non-deterministic behavior in oscillatorily sheared suspensions of non-interacting particles at high Reynolds numbers, Computers \& Fluids, 184, 78-90. [57] Lappa M. (2009), Thermal Convection: Patterns, Evolution and Stability, 700 pages, John Wiley \& Sons, Ltd (2009, Chichester, England).

[58] Lappa M., (2018), On the transport, segregation and dispersion of heavy and light particles interacting with rising thermal plumes, Physics of Fluids, 30(3), 033302 (23 pages).

[59] Maxey M. R. and Riley J. J., (1983), Equation of motion for a small rigid sphere in a nonuniform flow, Phys. Fluids, 26, 883-889.

[60] Kuhlmann H.C. et al. (2014), The JEREMI-Project on thermocapillary convection in liquid bridges. Part A: Overview of Particle Accumulation Structures, Fluid Dyn. Mater. Process., 10 (1), 1-36.

[61] Monti R., Savino R., Lappa M., (1998), Microgravity sensitivity of typical fluid physics experiment, presented at the 17 th Microgravity Measurements Group Meeting, Cleveland, Ohio, March 1998, published in the meeting proceedings in NASA CP-1998-208414, (23), 1-15.

[62] Monti R., Savino R., Lappa M., (2001), On the convective disturbances induced by g-jitter on the space station, Acta Astronautica, 48(5-12), 603-615.

[63] Savino R. and Lappa M., (2003), Assessment of the thermovibrational theory: application to gjitter on the Space-station, Journal of Spacecraft and Rockets, 40 (2), 201-210.

[64] Gershuni G.Z., Zhukhovitskii E.M., Yurkov Yu. S., (1982), Vibrational thermal convection in a rectangular cavity, Izv. Akad. Nauk SSSR Mekh. Zhidk. Gaza, 4, 94-99. 
[65] Gershuni G.Z., Zhukhovitskii E.M., (1986), Vibrational thermal convection in zero gravity, Fluid. Mech. Sov. Res., 15(1), 63-84.

[66] Clift R, Grace J.R, Weber M.E., (1978), Bubbles, Drops, and Particles, Academic Press, New York.

[67] Gresho P.M., Sani R.T., (1987), On pressure boundary conditions for the incompressible Navier-Stokes equations, Int. J. Numer. Meth. Fluids, 7, 1111-1145.

[68] Karniadakis G.E., Israeli M., Orszag S.A., (1991), High-Order Splitting Methods for the Incompressible Navier-Stokes Equations, Journal of Computational Physics, 97, 414-443.

[69] Petersson N.A., (2001), Stability of Pressure Boundary Conditions for Stokes and NavierStokes Equations, J. Comput. Phys., 172, 40-70.

[70] Ladyzhenskaya O.A., (1969), The Mathematical Theory of Viscous Incompressible Flow, Gordon and Breach, 2nd Edition, New York - London, 1969.

[71] Brown D.L., Cortez R. and Minion M.L., (2001), Accurate projecion methods for the incompressible Navier-Stokes equations, J. Comput. Phys., 168 (2), 464-499.

[72] Armfield S. and Street R., (2002), An analysis and comparison of the time accuracy of fractional-step methods for the Navier-Stokes equations on staggered grids, Int. J. Numer. Meth. Fluids, 38(3), 255-282.

[73] Guermond J.-L., Minev P. and Shen J., (2006), An Overview of Projection Methods for Incompressible Flows. Comput. Methods, Comput. Methods Appl. Mech. Eng., 195, 6011-6045.

[74] Patankar S., (1980), Numerical Heat Transfer and Fluid Flow (Hemisphere Series on Computational Methods in Mechanics and Thermal Science), Taylor \& Francis (1980).

[75] Lappa M., (1997), Strategies for parallelizing the three-dimensional Navier-Stokes equations on the Cray T3E, Science and Supercomputing at CINECA, 11, 326-340.

[76] Lappa M., (2019), A mathematical and numerical framework for the simulation of oscillatory buoyancy and Marangoni convection in rectangular cavities with variable cross section, Chapter 12 (pp. 419-458) in Computational Modeling of Bifurcations and Instabilities in Fluid Mechanics (Springer Mathematical Series, 2018, Part of the Computational Methods in Applied Sciences book series - COMPUTMETHODS, volume 50), Editor Alexander Gelfgat - ISBN 978-3-319-91493-0. DOI: 10.1007/978-3-319-91494-7.

[77] Khallouf H., Gershuni G. Z. and Mojtabi A., (1995), Numerical study of two-dimensional thermo-vibrational convection in rectangular cavities, Num. Heat Trans. A, 27, 297-305.

[78] Lappa M., (2011), "Some considerations about the symmetry and evolution of chaotic Rayleigh-Bénard convection: The flywheel mechanism and the "wind” of turbulence”, Comptes Rendus Mécanique, 339, 563-572. 